\title{
Two Protein N-Acetylgalactosaminyl Transferases Regulate Synaptic Plasticity by Activity-Dependent Regulation of Integrin Signaling
}

\author{
Neil Dani, ${ }^{1,2,3,4}$ He Zhu, ${ }^{1,2,3,4}$ and Kendal Broadie ${ }^{1,2,3,4}$ \\ Departments of ${ }^{1}$ Biological Sciences, ${ }^{2}$ Cell and Developmental Biology, and ${ }^{3}$ Pharmacology and ${ }^{4}$ The Vanderbilt Kennedy Center for Research on Human \\ Development, Vanderbilt University, Nashville, Tennessee 37232
}

\begin{abstract}
Using a Drosophila whole-genome transgenic RNAi screen for glycogenes regulating synapse function, we have identified two protein $\alpha$ - $N$-acetylgalactosaminyltransferases (pgant3 and pgant35A) that regulate synaptic 0 -linked glycosylation (GalNAc $\alpha 1-0-\mathrm{S} / \mathrm{T})$. Loss of either pgant alone elevates presynaptic/postsynaptic molecular assembly and evoked neurotransmission strength, but synapses appear restored to normal in double mutants. Likewise, activity-dependent facilitation, augmentation, and posttetanic potentiation are all suppressively impaired in pgant mutants. In non-neuronal contexts, pgant function regulates integrin signaling, and we show here that the synaptic Position Specific 2 ( $\alpha$ PS2) integrin receptor and transmembrane tenascin ligand are both suppressively downregulated in pgant mutants. Channelrhodopsin-driven activity rapidly ( $<1 \mathrm{~min})$ drives integrin signaling in wild-type synapses but is suppressively abolished in pgant mutants. Optogenetic stimulation in pgant mutants alters presynaptic vesicle trafficking and postsynaptic pocket size during the perturbed integrin signaling underlying synaptic plasticity defects. Critically, acute blockade of integrin signaling acts synergistically with pgant mutants to eliminate all activity-dependent synaptic plasticity.
\end{abstract}

Key words: activity dependent; Drosophila; integrin; neuromuscular junction; 0-linked glycosylation; plasticity

\section{Introduction}

The heavily glycosylated transmembrane and extracellular synaptomatrix at the synaptic interface plays pivotal roles in synaptogenesis, neurotransmission, and synaptic plasticity (Dityatev and Schachner, 2003; Broadie et al., 2011; Dani and Broadie, 2012). Neurological disease states arising from aberrant glycosylation occur in numerous congenital disorders of glycosylation and dystroglycanopathies (Freeze, 2006). However, the mechanisms by which synaptomatrix glycan modifications regulate normal synapse function and dysfunction in heritable disease states remain poorly understood (Ohtsubo and Marth, 2006). Drosophila is a powerful genetic model to pursue these synaptic glycan mechanisms, given the conservation of glycan pathways, reduced glycogene genomic redundancy in this system, and host of techniques available at the well characterized glutamatergic neuromuscular synapse (Keshishian et al., 1996; Gagneux and

Received April 11, 2014; revised July 23, 2014; accepted Aug. 11, 2014.

Author contributions: N.D. and K.B. designed research; N.D. and H.Z. performed research; N.D. and H.Z. analyzed data; N.D. and K.B. wrote the paper.

This work was fully supported by National Institute of Mental Health Grant MH096832 (K.B.). We are particularly grateful to Kelly Ten Hagen for pgant mutant and transgenic lines (pgant $3^{m 1}{ }^{\text {, pgant } 3^{m 2}}$, pgant $35 A^{H G 8}$, pgant $35 A^{3775}$, UAS-pgant3, and UAS-pgant35A), Zhuoren Wang for the optogenetic line (UAS-ChIEF-tdTomato) and the Bloomington Drosophila Stock Center for providing other essential stocks. We also thank the following for essential antibodies: Ron Wides (Ten-m), John Fessler (Tig), Talila Volk (LanA, Tsp), Stephan Baumgartner (Wb-N), Richard Hynes $\left(\beta_{\nu}\right)$, and the lowa Hybridoma Bank.

Correspondence should be addressed to Kendal Broadie, Vanderbilt University Station B, Box 35-1634, Nashville, TN 37235-1634. E-mail: kendal.broadie@vanderbilt.edu.

DOI:10.1523/JNEUROSCI.1484-14.2014

Copyright $\odot 2014$ the authors $\quad 0270-6474 / 14 / 3413047-19 \$ 15.00 / 0$
Varki, 1999). Using this model, we have shown recently that endogenous glycan-binding lectin [ mind the gap ( $\mathrm{mtg}$ ); Rushton et al., 2009], heparan sulfate proteoglycan (HSPG) modifiers [heparan sulfate 6-O-endosulfatase (sulf1)/heparan sulfate 6-O-sulfotransferase (hs6st); Dani et al., 2012], and N-linked glycosylation [UDP-GlcNAc: $\alpha$-3-D-mannoside- $\beta 1,2-N$-acetylglucosaminyltransferase I (mgat1); Parkinson et al., 2013] glycan mechanisms all act as potent regulators of trans-synaptic integrin, WNT (wingless-type MMTV integration site family), and bone morphogenetic protein (BMP) signaling.

To systematically pursue synaptic glycan mechanisms, we undertook a Drosophila whole-genome screen of glycogenes using RNAi-mediated knockdown of all N-/O-/glycosaminoglycanlinked enzymes, glycosyltransferases, and glycan-binding lectins, characterizing effects on neuromuscular junction (NMJ) structure and function using confocal microscopy and two-electrode voltage-clamp (TEVC) electrophysiology, respectively (Dani et al., 2012). This screen identified two $\alpha$-N-acetylgalactosaminyltransferases, pgant3 and pgant35A, that catalyze transfer of $\mathrm{N}$-acetylgalactosamine (GalNAc) monosaccharides onto serine/ threonine residues (GalNAc $\alpha 1-\mathrm{O}-\mathrm{S} / \mathrm{T}$ ) to form Tn antigens, as found within mucin-like O-linked glycans (Ten Hagen et al., 2003a). This most complexly regulated glycosylation is orchestrated by multiple GalNAc transferases (12 pgants in Drosophila) with distinct and overlapping peptide specificities (Yoshida et al., 2008; Tran and Ten Hagen, 2013). Pursuing our screen results with well characterized pgant 3 and pgant $35 \mathrm{~A}$ loss-of-function mutants (see Materials and Methods), we found elevated synaptic 
O-linked glycosylation, presynaptic/postsynaptic molecular assembly, presynaptic/postsynaptic ultrastructural elaborations, and neurotransmission strength, which are all corrected in double mutants that show none of these synaptic defects, identifying a novel suppressive genetic interaction.

In non-neuronal tissues, Drosophila pgants regulate integrin signaling and intercellular adhesion (Zhang and Ten Hagen, 2011). Importantly, we have shown that position-specific (PS) integrins, localized both presynaptically and postsynaptically, regulate NMJ morphogenesis (Beumer et al., 1999), synaptic scaffold/synaptomatrix adhesion molecules (Beumer et al., 2002), functional differentiation (Rohrbough et al., 2007), and activity-dependent plasticity (Rohrbough et al., 2000). Therefore, we hypothesized that pgants regulate integrin signaling at the synapse and consistently find suppressive downregulation of $\alpha$ PS2-containing integrin receptors (Beumer et al., 1999), RGDcontaining tenascin (Ten-m) ligand (Mosca et al., 2012), and postsynaptic membrane adhesion defects in pgant mutants. Furthermore, we find integrin- and activity-dependent functional synaptic plasticity is suppressively regulated in pgant mutants. Importantly, we find that channelrhodopsin activity stimulation (Wang et al., 2011) disrupts downstream integrin association with Talin and pFAK signaling and elevates postsynaptic membrane adhesion defects. RGD peptide blockade of integrin function synergistically abolishes all activity-dependent synaptic plasticity in pgant mutants. These data show that two pgants suppressively regulate synaptic O-GalNAc glycosylation, synapse molecular assembly, neurotransmission strength, and activity-dependent plasticity via trans-synaptic integrin-tenascin signaling.

\section{Materials and Methods}

Drosophila genetics. All stocks were maintained at $25^{\circ} \mathrm{C}$ on standard food. Two independent mutant alleles isolated by ethyl methanesulfonate (EMS) mutagenesis were used for pgant3: (1) pgant $3^{m 1}$, a $\mathrm{C}>\mathrm{T}$ transition changing conserved arginine to cysteine at amino acid 130 resulting in failure to glycosylate substrates in enzymatic activity tests; and (2) pgant $3^{m 2}, a \mathrm{G}>\mathrm{A}$ transition that creates a stop codon at amino acid 609, thereby deleting the C-terminal 59 aa and resulting in an unstable protein (Zhang et al., 2010). Similarly, the pgant35A mutations used included the following: (1) pgant $35 A^{H G 8}$, a $\mathrm{C}>\mathrm{T}$ transition at nucleotide 265 resulting in a glutamine to stop codon change at amino acid 89; and (2) pgant $35 A^{3775}$, a T $>$ A transversion at nucleotide 584 resulting in a premature stop codon at amino acid 195, both fully eliminating the catalytic domain (Ten Hagen and Tran, 2002). All mutants were placed in the $w^{1118}$ genetic background, and $w^{1118}$ was therefore used as the wild-type control. Rescue and overexpression experiments were performed with UAS-pgant3 and UAS-pgant35A (Zhang et al., 2008) wild-type transgenes driven by neural (elav-gal4; Lin and Goodman, 1994), muscle (24B-gal4; Brand and Perrimon, 1993), and ubiquitous (UH1-gal4; Wodarz et al., 1995) drivers. Standard genetic techniques were used to generate recombinant and multiply mutant animals. Optogenetic studies were performed with the UAS-ChIEF-tdTomato channelrhodopsin (chimera with a crossover site at loop E-F and isoleucine 170 mutated to valine) transgene (Wang et al., 2011) driven by the neural-specific elav-gal4 driver in animals raised on $0.25 \mathrm{~mm}$ all-trans retinal (Sigma) supplemented food. Animals used for experimentation were of either sex.

Immunocytochemistry. Wandering third instars were dissected in $\mathrm{Ca}^{2+}$-free saline and then fixed in $4 \%$ paraformaldehyde for $10 \mathrm{~min}$. Preparations were then washed in either permeabilizing PBST (PBS + $0.1 \%$ Triton X-100) or detergent-free PBS for extracellular labeling (Rushton et al., 2009). O-GalNAc glycans were visualized with TRITCconjugated vicia villosa lectin (VVA; 1:250; EY Laboratories) and helix pomatia lectin (HPL; 1:250; Invitrogen) (Chia et al., 2014). Mouse antibodies obtained from the Developmental Studies Hybridoma Bank included anti- $\beta$ PS (1:500), anti- $\alpha$ PS1 (1:200), anti- $\alpha$ PS2 (1:500), anti-scab
(1:200), anti-Talin (1:10), and anti-Disc large (DLG; 4F3; 1:250). Other sourced primary antibodies included the following: mouse anti-Ten-m (1:3000; Levine et al., 1994), mouse anti-Tiggrin (Tig; 1:200; Fogerty et al., 1994), guinea pig anti-LanA (1:200; Inoue and Hayashi, 2007), rat anti-Thrombospondin (Tsp; 1:200; Subramanian et al., 2007), rabbit anti-Wing-blister N-terminus (Wb-N; 1:500; Martin et al., 1999), rabbit anti- $\beta \nu$ (1:300; Yee and Hynes, 1993), and rabbit anti-pFAK (pY397; 1:50; Invitrogen). All antibodies were incubated at $4^{\circ} \mathrm{C}$ overnight. Alexa Fluor-647-conjugated goat anti-HRP and secondary antibodies (Jackson ImmunoResearch) were incubated at 1:250 for $2 \mathrm{~h}$ at room temperature.

Image quantification. Control and mutant preparations for antibody and lectin studies were processed simultaneously for all intensity comparisons (Dani et al., 2012). To allow for direct comparisons of signal intensity levels, all genotypes were dissected, fixed, labeled, and imaged in parallel at the same time, with identical confocal settings and intensity measurements also made at the same time for all compared genotypes. Imaging was done on an upright Zeiss LSM 510 META laser-scanning confocal using a Plan Apo 63× oil-immersion objective. NMJ structural quantification was done with anti-HRP imaging at muscle 6/7 in segment A3. All intensity analyses were done with NIH ImageJ software using the threshold function to outline $Z$-stack areas with the maximum projection function. All statistical comparisons were performed with one-way ANOVA analysis, followed by Dunnett's or Dunn's post hoc test for nonparametric data using Instat software (GraphPad Software). All data are presented as mean \pm SEM. All images were projected in LSM Image Examiner (Zeiss) and exported to Adobe Photoshop.

Electrophysiology. TEVC records were made from NMJs of paired control and mutant wandering third instars as reported previously (Beumer et al., 1999). Briefly, recordings were performed in the following solution (in mM): $128 \mathrm{NaCl}, 2 \mathrm{KCl}, 4 \mathrm{MgCl}_{2}, 1.0 \mathrm{CaCl}_{2}, 70$ sucrose, and 5 HEPES saline, $\mathrm{pH}$ 7.1. Recording electrodes ( $1 \mathrm{~mm}$ outer diameter capillaries; World Precision Instruments) filled with $3 \mathrm{M} \mathrm{KCl}$ had resistances of $>15$ $\mathrm{M} \Omega$. Evoked excitatory junction currents (EJCs) were recorded at $18^{\circ} \mathrm{C}$ using episodic recording from voltage-clamped $\left(V_{\text {hold }},-60 \mathrm{mV}\right)$ muscle 6 in segment A3 with a TEVC amplifier (Axoclamp 2B; Molecular Devices). Excitatory junctional potentials (EJPs) were also recorded in parallel. Segmental nerves were stimulated with a glass suction electrode at a suprathreshold voltage ( $50 \%$ above threshold) for $0.5 \mathrm{~ms}$ duration at 0.5 $\mathrm{Hz}$. For synaptic plasticity studies, the nerve was stimulated at $10 \mathrm{~Hz}$ for $60 \mathrm{~s}$ in $0.2 \mathrm{mM} \mathrm{CaCl}_{2}$ saline (Rohrbough et al., 2000). EJCs were acquired via Clampex (Molecular Devices) and analyzed using Clampfit 9.0 by averaging 10 [during initial/posttetanic potentiation (PTP)] to 20 (during tetanus) consecutive responses. Gly-Arg-Gly-Asp-Ser-Pro (GRGDSP) integrin inhibition and Gly-Arg-Ala-Asp-Ser-Pro (GRADSP) control peptides (Sigma) were used at $0.2 \mathrm{~mm}$, incubated for $1 \mathrm{~h}$ at $18^{\circ} \mathrm{C}$. Statistical comparisons were done using one-way ANOVA analysis, followed by Dunnett's post hoc test with Instat software (GraphPad Software). Each $n=1$ represents a recording from a different animal. All data are presented as mean \pm SEM.

Electron microscopy. Ultrastructural analyses were performed as reported previously (Beumer et al., 1999). Briefly, staged third-instar preparations were fixed in $1.6 \%$ paraformaldehyde/ $2 \%$ glutaraldehyde $(20$ $\mathrm{min}$ ), washed in $1 \times \mathrm{PBS}(10 \mathrm{~min})$, and transferred to $2.5 \%$ glutaraldehyde in cacodylate buffer ( $12 \mathrm{~h}$ ) with washes in the same buffer (30 min). Preparations were postfixed in $1 \% \mathrm{OsO}_{4}$ in cacodylate buffer $(2 \mathrm{~h})$ and then dehydrated in an ethanol series, followed by propylene oxide (30 min). Segment A3 muscle 6/7 was dissected free of the preparations and separately embedded in Araldite resin. Ultrathin (40 nm) sections were cut using a Leica ultracut UCT 54 ultramicrotome and then transferred to Formvar-coated slot grids. Sections were imaged using a Phillips CM10 transmission electron microscope at $80 \mathrm{kV}$, with images collected on a 4 megapixel CCD camera. Sample sizes are $\geq 10$ independent NMJs, with the statistical analyses calculated using unpaired $t$ tests. Images acquired from AMT Image Capture Software were exported to Adobe Photoshop. All data are presented as mean \pm SEM.

Optogenetics. Wandering third instars were dissected in $0.2 \mathrm{mM} \mathrm{Ca}^{2+}$ saline on Sylgard-coated plates with the nervous system kept intact. An LEDD1B LED driver, M470L2 mounted LED at $470 \mathrm{~nm}$ affixed with an LA1951-A lens was used to stimulate channelrhodopsin activity (Gruntman and Turner, 2013). Preparations were subjected to a $60 \mathrm{~s}$ train of 
light stimulation at $10 \mathrm{~Hz}$, with a pulse duration of $60 \mathrm{~ms}$, followed by immediate fixation and processing during continual stimulation, using the methods described above. At least eight independent NMJs were analyzed for each genotype and condition, with statistical tests for activity-dependent changes in fluorescence intensity and ultrastructure performed as described above in the immunocytochemistry and electron microscopy sections.

\section{Results \\ pgants regulate synapse composition and transmission strength}

An unbiased genetic screen of glycogenes identified synaptic function defects using inducible RNAi-mediated downregulation of two pgants (pgant3 and pgant35A). This screen tested 130 glycan-related genes defined in eight function categories: $\mathrm{N}$-glycan, O-glycan, and glycosaminoglycan biosynthesis; glycosyltransferases, glycan degrading/modifying enzymes; glycoprotein and proteoglycan core proteins; and sugar transporters and glycan-binding lectins. Using a combination of confocal microscopy and TEVC electrophysiology, NMJ morphology and functional transmission defects were tested in Drosophila wandering third-instar larvae following ubiquitous (UH1-gal4) RNAi knockdown. From this screen, 31 genes affected synapse structure (27 increased bouton number, two increased branching, and two increased NMJ area), and 13 affected synapse function (12 increased and one decreased). Only six gene knockdowns affected both structure and function. To investigate mucin-type O-linked glycosylation, nine available RNAi lines were used to test six pgant genes (pgant2, pgant3, pgant4, pgant5, pgant6, and pgant35A) and three additional GalNAc transferases (GalNAcT-1, GalNAcT-2, and C1GalTA). Of these, three pgant genes (pgant3, pgant5, and pgant35A) were identified to have increased neurotransmission strength during knockdown, and GalNAc-T2 showed increased NMJ area. The other five gene knockdowns caused no detectable NMJ phenotypes. Well characterized mutants are available for only pgant 3 and pgant $35 \mathrm{~A}$ (see Materials and Methods), which have been studied extensively in heteroallelic null combinations (Ten Hagen and Tran, 2002; Zhang et al., 2010). In this study, we pursued a full characterization of these two pgant genes using the same conditions.

To characterize synaptic mechanisms in pgant 3 and pgant $35 \mathrm{~A}$ null single and double mutant larvae, we performed nerveevoked EJC recordings in the TEVC paradigm (Dani et al., 2012). Sample traces of 10 consecutive, superimposed responses are shown for four genotypes: the genetic background control $\left(w^{1118}\right)$, pgant3 $\left(\right.$ pgant $^{m 1} /$ pgant $\left.^{m 2}\right)$ and pgant $35 A$ (pgant $35 A^{H G 8} /$ pgant $^{2} 3 A^{3775}$ ) single mutants in $w^{1118}$ background, and the double null mutant ( pgant $3^{m 1}$,pgant $35 A^{H G 8} /$ pgant $3^{m 2}$,pgant $35 A^{3775}$ ). Neurotransmission is clearly and consistently elevated in both pgant mutants, increased 25-40\% compared with controls (Fig. 1A). Quantification of mean EJC amplitudes shows that synapse strength is very significantly elevated in both pgant3 (255.46 \pm $8.12 \mathrm{nA}, n=26, p<0.001)$ and pgant35A (277.62 $\pm 11.88 \mathrm{nA}$, $n=22, p<0.001)$ single mutants compared with control (198.73 $\pm 7.77 \mathrm{nA}, n=17$; Fig. $1 A$, right). Surprisingly, however, neurotransmission in the recombinant double null mutant is not significantly elevated compared with control $(231.64 \pm 7.24 \mathrm{nA}$, $n=21, p>0.05$; Fig. $1 A$, right), which behaves like the control. Thus, a similar phenotype occurs in the two pgant single mutants, which is absent in the double mutant. We use the term "suppression" throughout this study, as the simplest genetic term describing the observed interaction. Importantly, there is a synaptic function defect only, with no differences in NMJ morphology in either of the pgant mutants. In quantifying synaptic branching, neither pgant3 $(5.00 \pm 0.28$ decrease, $n=28)$ nor pgant $35 \mathrm{~A}$ $(5.79 \pm 0.26, n=24)$ single mutants or the double mutants $(6.26 \pm 0.41, n=23)$ showed any significant difference from controls $(5.58 \pm 0.22, n=26)$. Likewise, NMJ bouton number is also not significantly affected in pgant3 $(90.89 \pm 4.25, n=28)$ or pgant35A (92.29 $\pm 5.78, n=24)$ single mutants or double mutants $(77.46 \pm 4.18, n=24)$ compared with controls $(85.96 \pm$ $4.18, n=28$ ). This finding was the first discovery of the suppressive action of pgant genes on synapse function.

To begin to determine how pgant corepressive regulation arises at the synapse, we labeled NMJs for presynaptic active zones with Bruchpilot (Brp) and postsynaptic glutamate receptors (GluRIID), marking the two sides of each individual synapse (Fig. $1 B$ ). There is a clear and consistent increase in Brp/GluRIID punctae in both pgant 3 and pgant $35 \mathrm{~A}$ single mutants, indicating a cooperative change on both presynaptic and postsynaptic sides of the synapse. Importantly, however, the double null mutant does not show any detectable increase in either synaptic marker (Fig. $1 B)$. Quantification reveals significantly increased glutamate receptor field area and punctae number in pgant3 $[82.59 \pm 6.77$ $\mu \mathrm{m}^{2}(p<0.05)$ and $\left.358.0 \pm 16.20 \mu \mathrm{m}^{2}(p<0.01) ; n=14\right]$ and pgant35A $\left(81.02 \pm 6.95\right.$ and $302.73 \pm 15.61 \mu \mathrm{m}^{2} ; n=15, p<$ $0.05)$ single mutants but with no differences in the double mutants in either parameter compared with controls $(61.03 \pm 3.99$ and $233.25 \pm 12.33 \mu \mathrm{m}^{2} ; n=16$; Fig. 1C). Likewise, presynaptic Brp active zone area and punctae number are increased at pgant 3 $\left(54.80 \pm 4.80\right.$ and $\left.357.46 \pm 18.89 \mu \mathrm{m}^{2} ; n=13, p<0.05\right)$ and pgant35A (52.96 \pm 4.30 and $305.4 \pm 14.86 \mu \mathrm{m}^{2} ; n=15, p<$ $0.05)$ single mutants, with no differences in pgant3,pgant $35 A$ double mutants compared with controls (38.56 \pm 3.03 and $242 \pm$ $31.22 \mu \mathrm{m}^{2} ; n=16$; Fig. $1 C$ ). Both pgant mutants show no significant change in spontaneous miniature EJC (mEJC) frequencies (pgant3, $2.29 \pm 0.17 \mathrm{~Hz}, n=10$; pgant35A, $2.06 \pm 0.11 \mathrm{~Hz}, n=$ 9) compared with control $(1.93 \pm 0.13 \mathrm{~Hz}, n=10)$ but do show small, significant decreases in mEJC amplitudes (pgant3, $0.68 \pm$ $0.03 \mathrm{nA}, n=10, p<0.05$; pgant35A, $0.69 \pm 0.03 \mathrm{nA}, n=9, p<$ $0.05)$ compared with control $(0.80 \pm 0.02 \mathrm{nA}, n=10)$. These results show that pgant 3 and pgant $35 A$ both upregulate neurotransmission strength through elevated presynaptic and postsynaptic assembly via a mutually suppressive mechanism that predominantly affects evoked function.

\section{pgants regulate presynaptic vesicles and postsynaptic pocket size}

The synaptic ultrastructure of the Drosophila NMJ has been well characterized by transmission electron microscopy (TEM), categorizing multiple synaptic vesicle (SV) pools in the presynaptic bouton and the complex architecture of the expansive subsynaptic reticulum (SSR) of the postsynaptic membrane (Rohrbough et al., 2007). Because no gross morphology differences were associated with observed neurotransmission elevations in pgant mutants, we next investigated synapse ultrastructure. On the presynaptic side, we measured bouton area, active zone architecture, overall SV density, and SV distribution in concentric rings (e.g., $250 \mathrm{~nm}, 500 \mathrm{~nm}$ ) extending from each active zone (Fig. $2 A, B$; white arrows). On the postsynaptic side, we assayed SSR area, thickness on major and minor axes, density (folds/unit length), and postsynaptic pocket (PSP) size by measuring the distance from the presynaptic active zone to the first SSR membrane layer (Fig. 2A, B; dotted white lines). Expansion of the PSP is a hallmark of mutants defective in synaptomatrix resident, trans- 

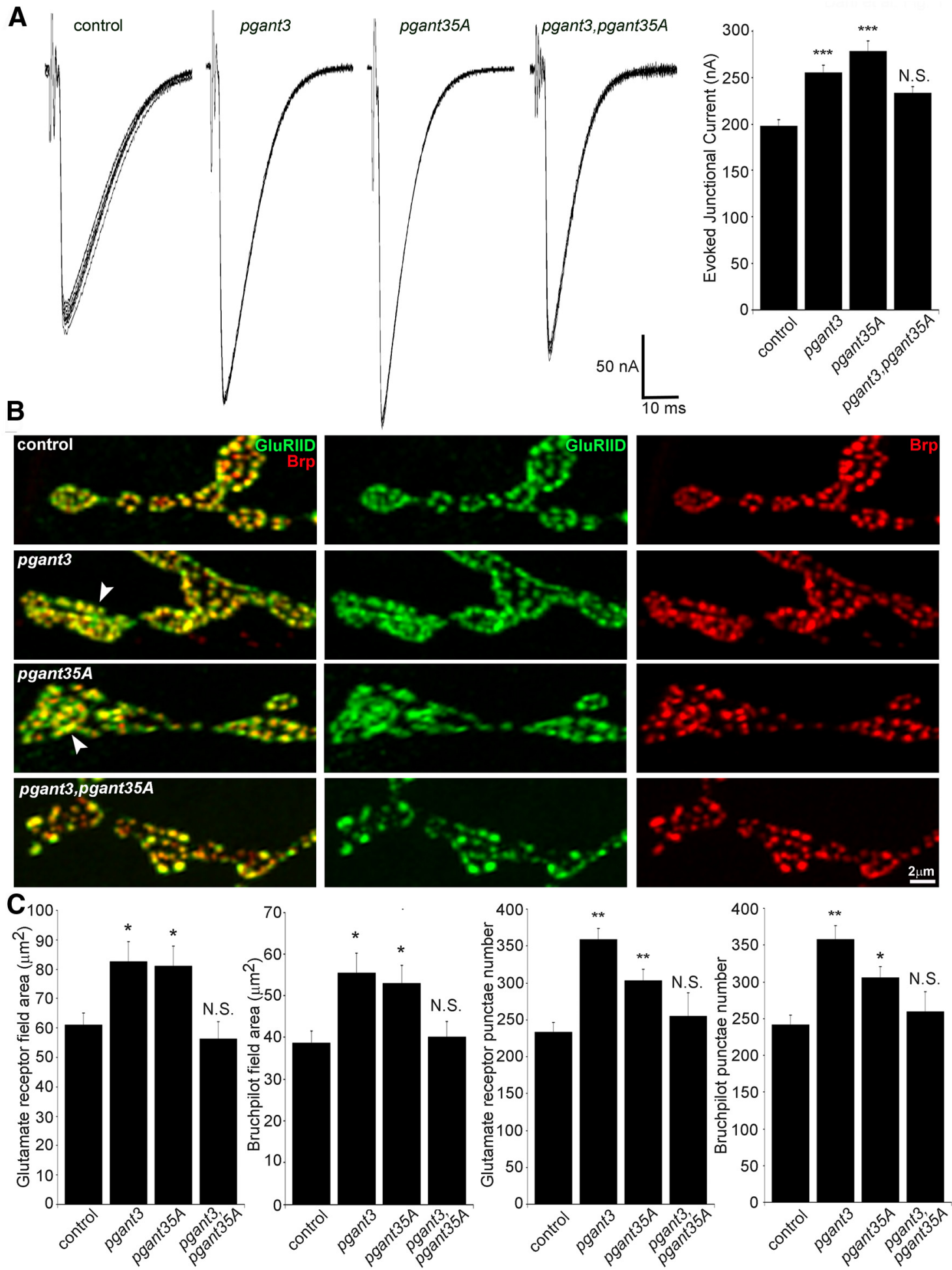

Figure 1. Null pgant mutants suppressively elevate neurotransmission strength. $A$, Representative evoked EJC records from genetic control $\left(w^{1118}\right)$, pgant3 $\left(p g a n t 3^{m 1} / p g a n t 3^{m 2}\right)$ and $p g a n t 35 A$

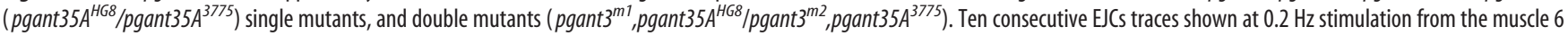
$\mathrm{NMJ}$ in segment A3. Calibration: $10 \mathrm{~ms}, 50 \mathrm{nA}$. Right, Histogram of mean EJC amplitudes, with sample sizes $\geq 17$ for each genotype. $\boldsymbol{B}$, Representative NMJ boutons for the above four genotypes labeled for postsynaptic glutamate receptors (GluRIID, green) and presynaptic active zone Brp (red), with split channels shown for clarity. White arrows indicate GluRIID/Brp paired punctae in single mutants. Scale bar, $2 \mu \mathrm{m}$. C, Quantification of GluRIID/Brp areas and punctae number per NMJ terminal. Statistical differences are calculated using one-way ANOVA with Dunnett's post hoc test: ${ }^{*} p<0.05,{ }^{* *} p<0.01,{ }^{* * *} p<0.001$. N.S., No significance. Error bars indicate SEM.

synaptic signaling ligands (Packard et al., 2002) and HSPG extracellular regulators of trans-synaptic signaling (Kamimura et al., 2013).

Presynaptic bouton appearance (Fig. 2A) and area in both pgant mutants (pgant3, $7.53 \pm 0.85 \mu \mathrm{m}^{2}, n=10$; pgant $35 \mathrm{~A}$, $\left.7.76 \pm 0.80 \mu \mathrm{m}^{2}, n=12\right)$ are not significantly different from the genetic control $\left(w^{1118}, 9.51 \pm 1.78 \mu \mathrm{m}^{2}, n=7\right)$, although there is a trend toward smaller boutons. Likewise, active zone architecture and t-bar dimensions are not measurably affected by loss of 
A
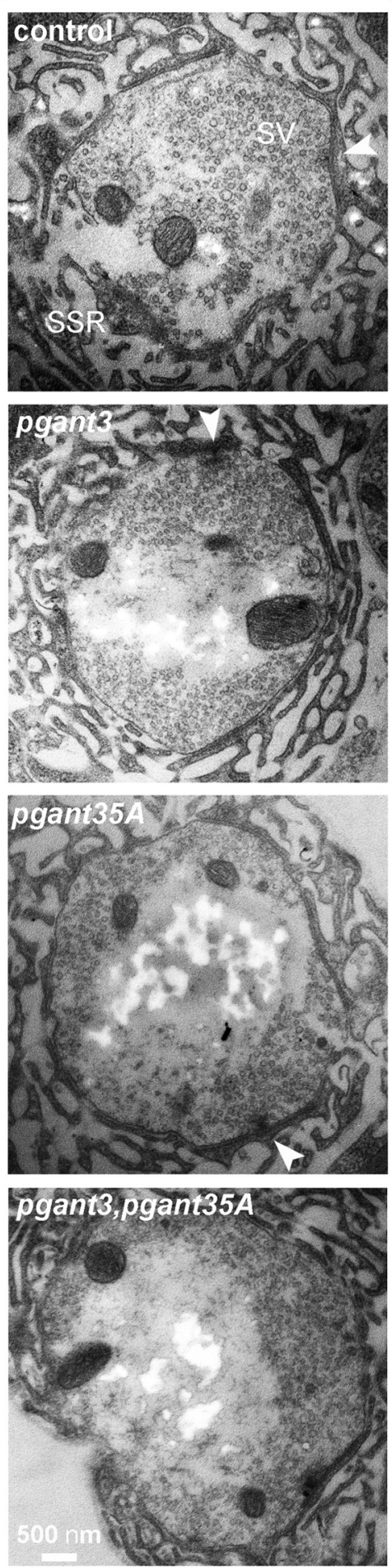

B
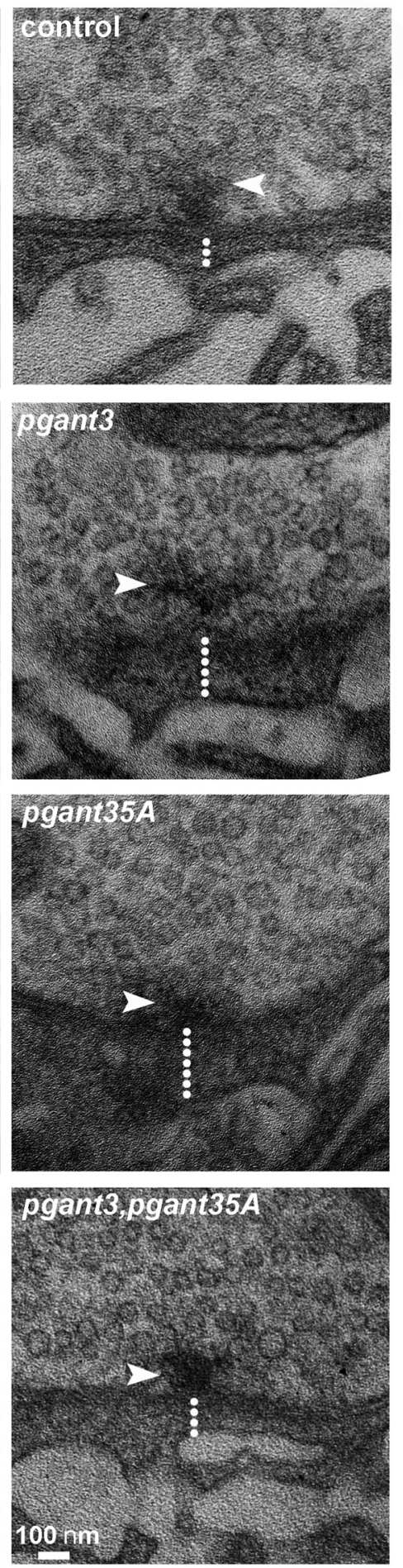

C
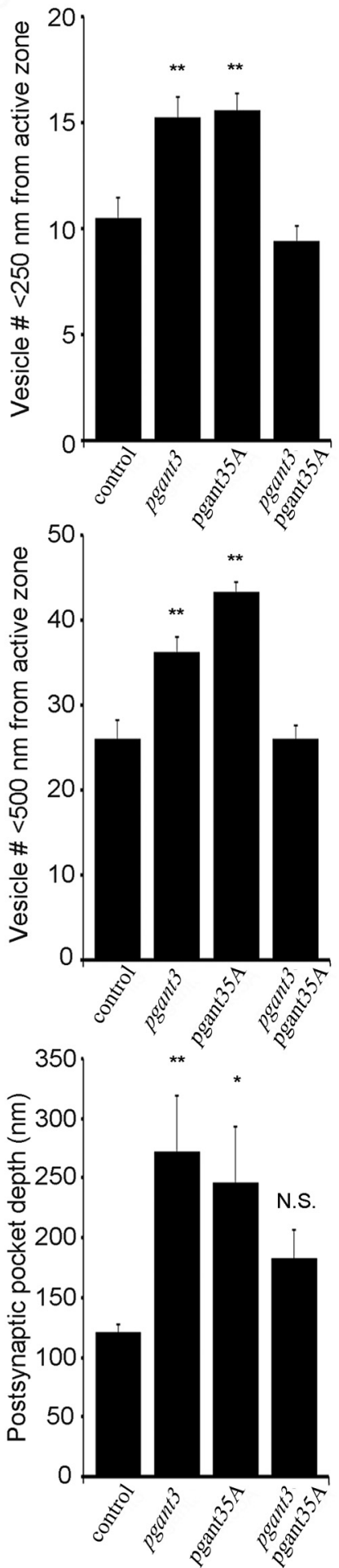

Figure 2. Null pgant mutants suppressively alter presynaptic/postsynaptic ultrastructure. $A$, Representative TEM images of synaptic boutons from muscle 6 NMJ in segment $A 3$ of genetic control

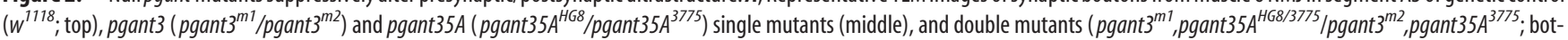
tom). Labels indicate SV pools in presynaptic bouton, SSR of muscle membrane, and presynaptic active zones (arrowheads). Scale bar, $500 \mathrm{~nm}$. $\boldsymbol{B}$, High-magnification images of single active zone synapses in all four genotypes. Labels indicate presynaptic t-bars (arrowheads) and PSPs (white dotted lines). Scale bar, $100 \mathrm{~nm}$. C, Quantification of SV pools [ <250 nm (top), < $500 \mathrm{~nm}$ (middle) from active zone t-bar] and PSP depth (bottom). Sample size is $\geq 10$ boutons for each of the four genotypes. Statistical differences calculated using one-way ANOVA with Dunnett's post hoc test: ${ }^{*} p<0.05,{ }^{* *} p<0.01$. N.S., Not significance. Error bars indicate SEM.

pgant activity (Fig. 2B). In contrast, the density and distribution of SV pools is clearly aberrant in both pgant single mutants, although the double null mutant is not detectably different from the control (Fig. 2A,B). Immediately adjacent to active zone t-bars (Fig. $2 B$, arrows), SV clustering is increased in both pgant single mutants but not in the double mutant combination. Quantifying SV number within $250 \mathrm{~nm}$ of the t-bar shows a consistent density in controls $(10.5 \pm 0.91)$, which is significantly increased 
in both pgant3 $(15.22 \pm 0.99, p<0.01)$ and pgant $35 A(15.53 \pm$ $0.78, p<0.01)$ single mutants but back at the control level in double null mutants ( $9.4 \pm 0.67, n=15$; Fig. $2 C$, top). Likewise, at a distance of $500 \mathrm{~nm}$ from the active zone t-bar, SV number increases in pgant3 $(36.3 \pm 1.66, p<0.01)$ and pgant35A $(43.31 \pm 1.21, p<0.01)$ single mutants but not in double mutants $(26.06 \pm 1.44, n=15)$ compared with controls $(26 \pm 2.18$; Fig. $1 C$, middle). Thus, presynaptic vesicle pool distribution is suppressively regulated by pgant3 and pgant35A, in line with changes in synaptic function.

Postsynaptic SSR appearance, area, thickness, and density are normal in all pgant mutants (Fig. 2A). Quantification of normalized SSR area (pgant3, $1.26 \pm 0.16$; pgant35A, $1.13 \pm 0.17$; pgant3,pgant35A, $1.09 \pm 0.17$ ), thickness (pgant3, $1.12 \pm 0.10$; pgant35A, $0.84 \pm 0.08$; pgant3,pgant $35 A, 0.80 \pm 0.07)$, and normalized density (pgant3, $0.83 \pm 0.05$; pgant $35 A, 1.10 \pm 0.08$; pgant3,pgant35A, $1.11 \pm 0.08$ ) all show no significant changes normalized to controls (Fig. 2A). In contrast, however, there is a striking expansion in both pgant mutants of the PSP (Packard et al., 2002; Kamimura et al., 2013). This compartment has been defined as "a postsynaptic area immediately apposed to an active zone containing amorphous material” (Packard et al., 2002), which is spatially localized between the postsynaptic membrane and SSR (Ren et al., 2009). The PSP compartment has been shown to be expanded in trans-synaptic signaling disrupted mutants, including WNT wingless, BMP glass bottom boat, HSPG perlecan, and HSPG sulfateless mutants (Packard et al., 2002; Tian and Ten Hagen, 2007; Ren et al., 2009; Nahm et al., 2010; Kamimura et al., 2013). Both pgant3 and pgant35A single mutants similarly display an enlarged PSP compartment, although the double null mutant is not detectably different from the control (Fig. 2B, dotted white lines). As a quantifiable PSP parameter, pocket depth from the presynaptic active zone to the next adjacent postsynaptic SSR membrane was measured in all four genotypes. Compared with controls (mean PSP depth, $121.17 \pm 4.95$ nm, $n=10$ ), both pgant single mutants display a more than twofold expanded PSP (pgant3, $254.7 \pm 35.25 \mathrm{~nm}, p<0.01$; pgant35A, $233.96 \pm 41.83 \mathrm{~nm}, p<0.05$; Fig. $2 B, C$, bottom). In sharp contrast, the double null mutants show no significant increase in PSP depth compared with controls (169.09 $\pm 15.46 \mathrm{~nm}$, $n=12$; Fig. $2 C$, bottom). Thus, we observe suppressive regulation by pgant genes of SV pools in the presynaptic compartment as well as postsynaptic compartment expansion, paralleling the changes in neurotransmission strength.

\section{Neuronal and muscle pgant 3 and pgant $35 \mathrm{~A}$ modulate neurotransmission}

To determine cell-specific requirements of pgant 3 and pgant $35 \mathrm{~A}$, we used the inducible Gal4-UAS binary system (Brand and Perrimon, 1993) to express UAS-pgant3 or UAS-pgant35A wildtype transgenes in neurons (elav-gal4) or muscles $(24 B-$ gal4) in respective single mutant backgrounds and assayed for phenotype rescue (Fig. 3). Sample EJC traces show an average of 10 consecutive nerve-evoked responses for both rescue conditions in both pgant nulls (Fig. 3A). We find that functional neurotransmission strength is restored to control levels when pgant 3 is expressed in either neurons $\left(\right.$ pgant $3^{m 1} /$ pgant $3^{m 2}$;UAS-pgant3/elav, $181.61 \pm$ $11.85 \mathrm{nA}, n=14$ ) or muscles (pgant $3^{m 1} /$ pgant $^{m 2}$;UAS-pgant3/ $24 B, 187.47 \pm 12.72 \mathrm{nA}, n=11)$ in the otherwise pgant3 null background compared with controls $\left(w^{1118}, 193.34 \pm 8.69 \mathrm{nA}\right.$, $n=14$; Fig. $3 C$, top). Similarly, pgant $35 A$ expression in neurons (pgant35 $A^{H G 8} /$ pgant $35 A^{3775}$;UAS-pgant35A/elav, 197.50 $\pm 14.26 \mathrm{nA}, n=9)$ or muscles $\left(\right.$ pgant $35 A^{H G 8} /$ pgant $35 A^{3775} ; U A S-$ pgant $35 A / 24 B, 211.42 \pm 17.06 \mathrm{nA}, n=10)$ in the pgant $35 A \mathrm{mu}-$ tant background likewise rescues neurotransmission strength to control levels (Fig. 3C, top).

To test whether this functional rescue correlates with corrected synaptic molecular assembly, NMJs were labeled for presynaptic Brp and postsynaptic GluRIID (Fig. 3B). Brp punctae number in pgant3 neuronal $(392.56 \pm 22.86, n=9)$ or muscle $(321.13 \pm 17.84, n=15)$ rescue conditions and pgant35A neuronal $(340 \pm 25.63, n=8)$ or muscle $(409.58 \pm 27.71, n=12)$ rescue conditions does not differ significantly from control (358.84 $\pm 18, n=19$; Fig. $3 B, C$ ). Similarly, GluRIID punctae number is also restored to control levels $(360.73 \pm 19.16, n=19)$, with pgant3 neuronal $(395.89 \pm 23.95, n=9)$ or muscle (327.6 \pm $18.22, n=15)$ rescue and pgant35A neuronal $(336 \pm 24.71, n=$ 8 ) or muscle ( $414.17 \pm 26.98, n=12)$ rescue. The same result is reflected in Brp area measurements, in which pgant3 neuronal $(85.67 \pm 6.78, n=10)$ or muscle $(75.66 \pm 7.99, n=15)$ rescue and pgant35A neuronal (79.12 $\left.\pm 8.23 \mu \mathrm{m}^{2}, n=10\right)$ or muscle $\left(88.80 \pm 8.29 \mu \mathrm{m}^{2}, n=12\right)$ rescue is similar to control values (74.35 $\pm 4.69 \mu \mathrm{m}^{2}, n=18$; Fig. $\left.3 \mathrm{~B}, C\right)$. Similarly, postsynaptic GluRIID area measurements in pgant3 neuronal (173.75 \pm 10.38 $\left.\mu \mathrm{m}^{2}, n=10\right)$ or muscle $\left(142.47 \pm 10.80 \mu \mathrm{m}^{2}, n=15\right)$ rescue and pgant $35 \mathrm{~A}$ neuronal $\left(155.61 \pm 9.97 \mu \mathrm{m}^{2}, n=10\right)$ or muscle $\left(156.99 \pm 11.89 \mu \mathrm{m}^{2}, n=12\right)$ rescue are not significantly different from control $\left(148.20 \pm 7.01 \mu \mathrm{m}^{2}, n=18\right.$; Fig. $\left.3 C\right)$. These results show that both pgant 3 and pgant $35 \mathrm{~A}$ can function either presynaptic or postsynaptically to regulate synaptic assembly and neurotransmission strength.

\section{Presynaptic/postsynaptic balance of pgant 3 and pgant $35 \mathrm{~A}$ regulate neurotransmission}

Given the pgant suppressive mechanism and coupled presynaptic/postsynaptic roles of pgant 3 and pgant35A, we next tested whether the balance of pgant 3 and pgant $35 A$ is required to properly regulate neurotransmission. We generated allelic combinations for UAS-pgant3 wild-type transgene expression in neurons (elav-gal4), muscles (24B-gal4), or ubiquitously (UH1-gal4) in the pgant3,pgant35A double mutant background and tested effects on neurotransmission strength. Representative EJC traces for each genotype are shown in Figure $4 A$. Compared with the control mean EJC amplitude (pgant $3^{\text {m1 }}$,pgant $35 A^{H G 8}$ /

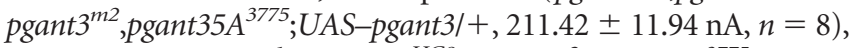
neuronal ( pgant $^{m 1}{ }^{m 1}$ pgant $35 A^{H G 8} /$ pgant $3^{m 2}$,pgant $35 A^{3775}$; elav/ UAS-pgant3, $258.99 \pm 9.59 \mathrm{nA}, n=9, p<0.05)$, muscle ( pgant3 ${ }^{m 1}$,pgant $35 A^{H G 8} /$ pgant $3^{m 2}$,pgant $35 A^{3775} ; 24 B / U A S-$ pgant3, $276.52 \pm 11.19 \mathrm{nA}, n=9, p<0.05)$, and ubiquitous ( pgant3 ${ }^{m 1}$,pgant $35 A^{H G 8} /$ pgant ${ }^{m 2}$,pgant $35 A^{3775}$;UH1/UAS-pgant3, $254.47 \pm 13.59 \mathrm{nA}, n=13, p<0.05)$ pgant 3 expression in the double mutant background all significantly elevated neurotransmission strength (Fig. $4 B$ ). Thus, restoring pgant 3 to either neuron or muscle effectively reveals the pgant $35 A$ single mutant phenotype (Fig. $1 A$ ).

In parallel, we overexpressed both pgant genes alone to test the effect on neurotransmission strength. Representative EJC traces for each genotype are shown in Figure 4C. Compared with control (UAS-pgant3/+, $205.55 \pm 8.77 \mathrm{nA}, n=22)$, pgant3 overexpression in neurons (UAS-pgant3/elav, $154.99 \pm 11.99 \mathrm{nA}$, $n=11, p<0.01$ ), muscles (UAS-pgant3/24B, $165.62 \pm 11.13 \mathrm{nA}$, $n=10, p<0.05$ ), or ubiquitous (UAS-pgant3/UH1, $164.80 \pm$ $9.79 \mathrm{nA}, n=10, p<0.05)$ all similarly decreased mean EJC amplitudes (Fig. 4D, left). Likewise, compared with control (UAS pgant35A/+, $235.15 \pm 10.77 \mathrm{nA}, n=19)$, pgant35A overexpression in neurons (UAS-pgant35A/elav, $193.50 \pm 13.39 \mathrm{nA}, n=18$, $p<0.05)$ and ubiquitous overexpression (UAS-pgant35A/UH1, 
A

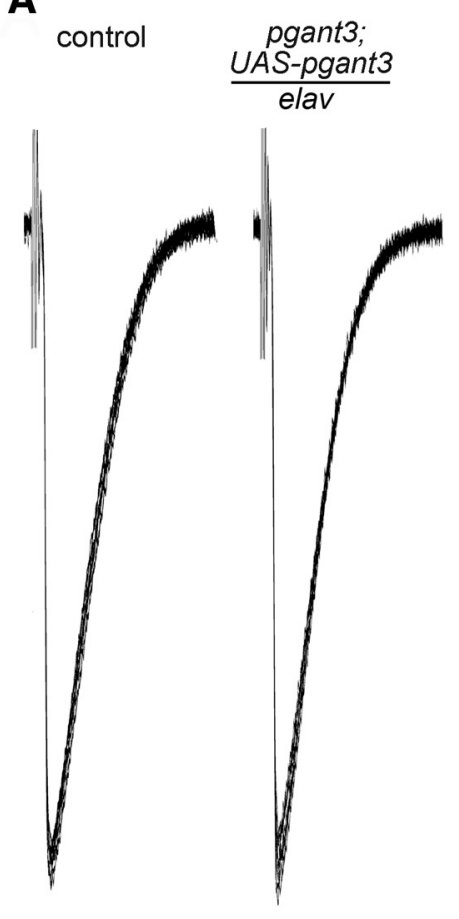

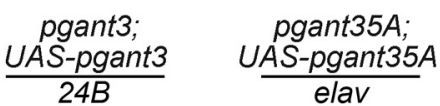

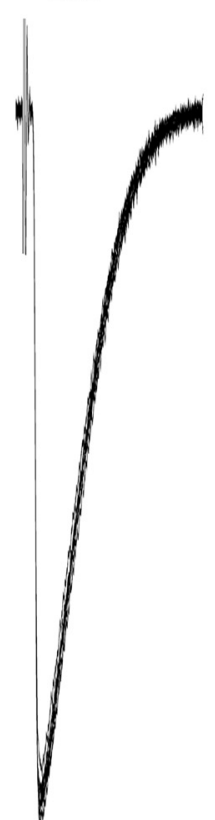

B
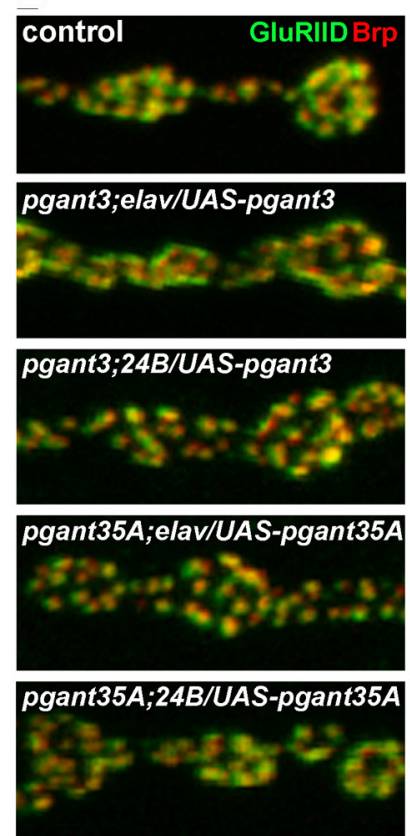
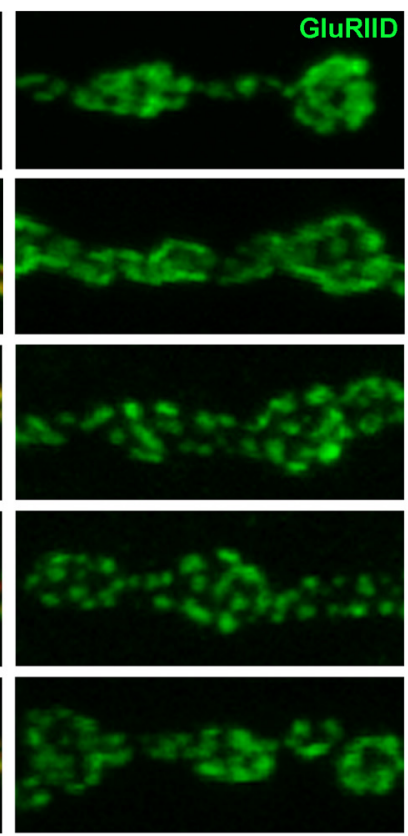

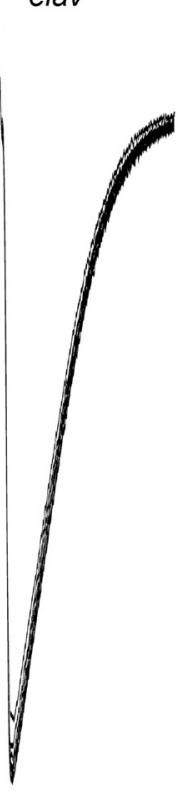

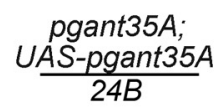
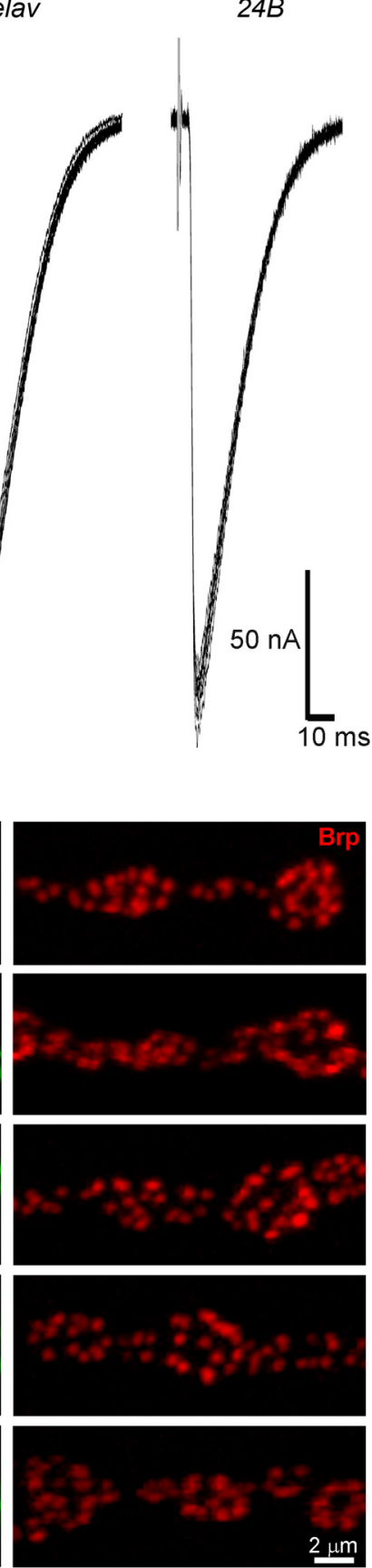

C
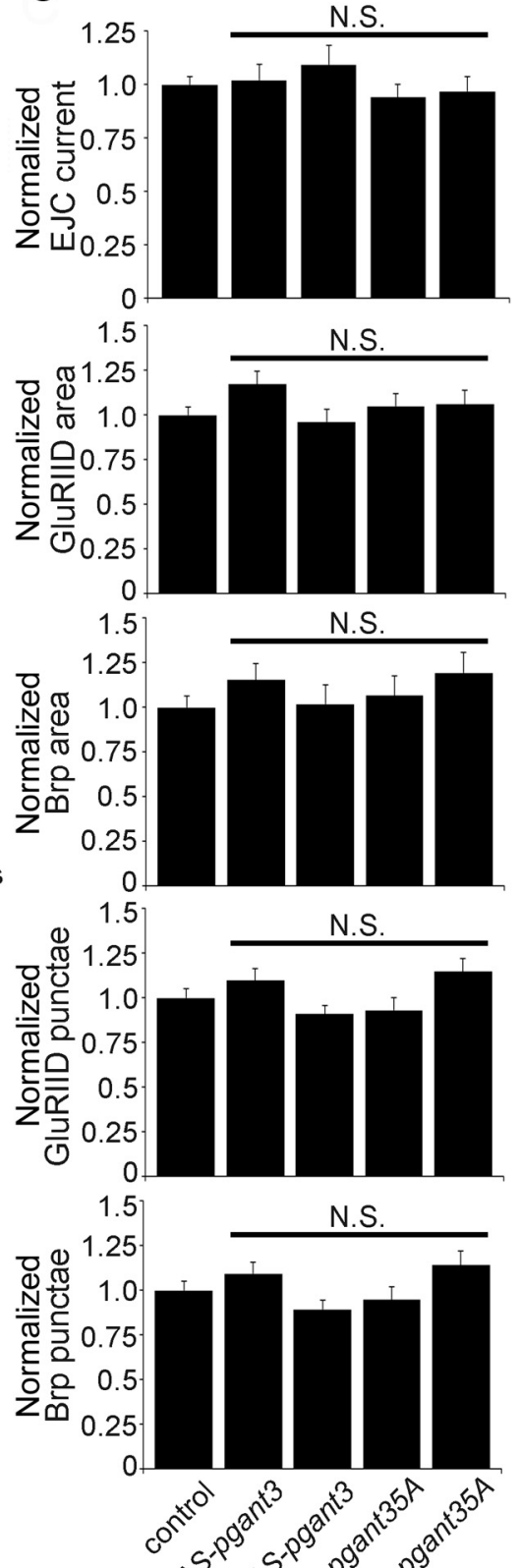

Figure 3. pgants function in neurons and muscle to regulate neurotransmission. $\boldsymbol{A}$, Representative EJC records from genetic control $\left(w^{1118}\right), p g a n t 3$ mutant with neuronal $\left(p g a n t 3^{m 1} / p g a n t 3^{m 2}\right.$;

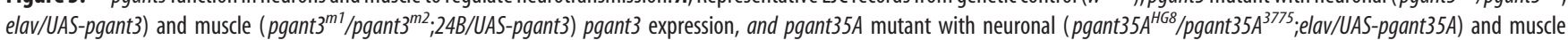

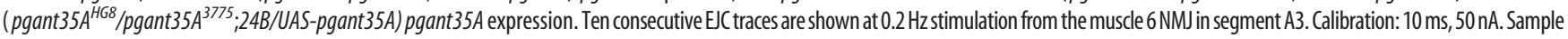
size is $n \geq 8$ animals for all conditions. $\boldsymbol{B}$, Representative NMJ boutons for the above five genotypes colabeled for both postsynaptic glutamate receptors (GluRIID; green) and presynaptic active zone Brp (red), with split channels shown for clarity. Scale bar, $2 \mu \mathrm{m}$. C, Histograms show normalized EJC amplitude, punctae number, and GluRIID/Brp area per NMJ terminal for the above five genotypes. Sample size is $n \geq$ 10 animals for all conditions. Statistical differences are calculated using one-way ANOVA with Dunnett's post hoc test. N.S. indicates no significance. Error bars indicate SEM.

$187.52 \pm 9.43 \mathrm{nA}, n=10, p<0.05)$ both decrease neurotransmission transmission, although muscle overexpression alone has no significant effect ( $U A S-$ pgant $35 A / 24 B, 224.99 \pm 8.77$ nA, $n=20$; Fig. $4 D$, right). Overall, pgant overexpression has the opposite consequence of pgant loss of function (Fig. $1 A$ ). Thus, the proper balance of pgant3/pgant $35 \mathrm{~A}$ in neurons and muscle bidirectionally regulates the strength of synaptic transmission.
Activity-dependent synaptic plasticity is impaired in pgant mutants

In the non-neuronal context of the Drosophila wing disc, pgant mutants specifically impair integrin signaling to cause intercellular de-adhesion (Zhang et al., 2010). Similarly, the above synaptic ultrastructure defects in pgant mutants recalls synaptic integrin signaling, which we have shown is required for activity-dependent 
A

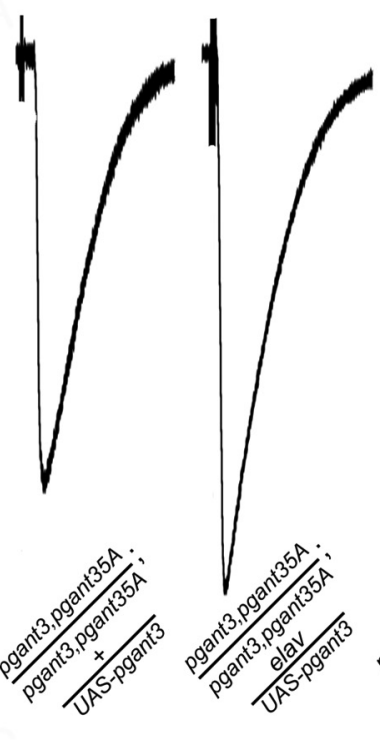

B
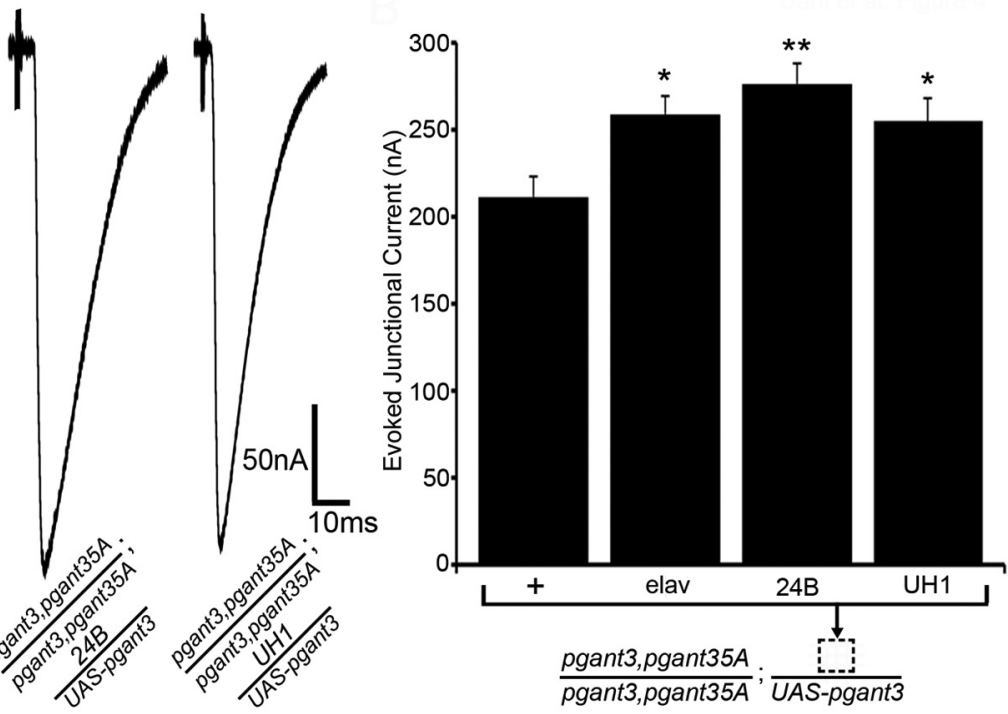

C
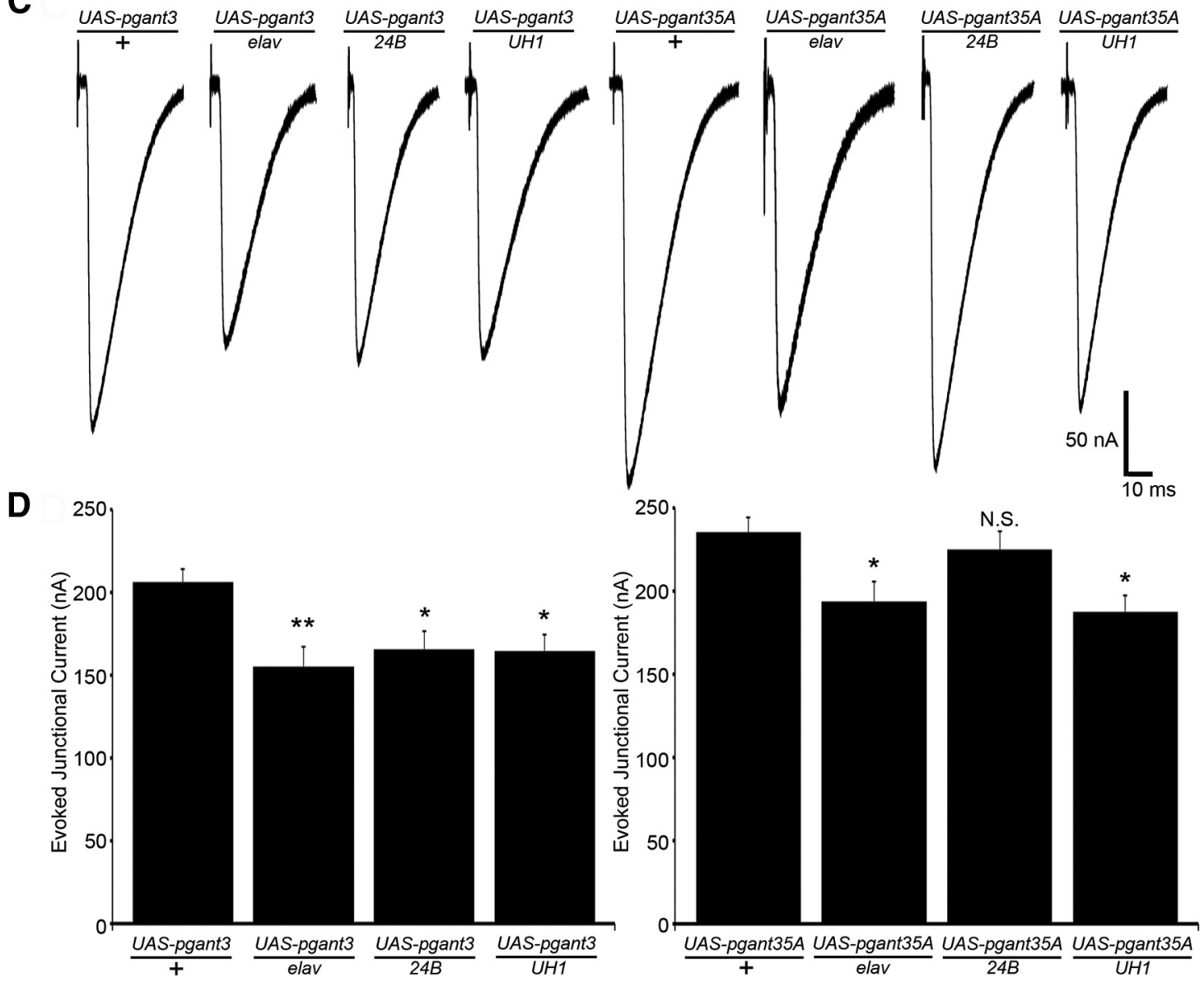

Figure 4. Pre/postsynaptic pgant $3 / 35 A$ balance regulates neurotransmission. Representative EJC records $(\boldsymbol{A})$ and mean amplitudes $(\boldsymbol{B})$ for pgant3 expression in the double mutant background

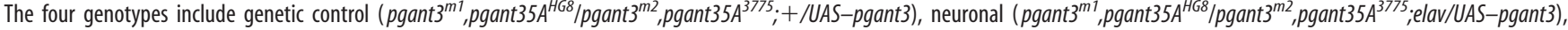

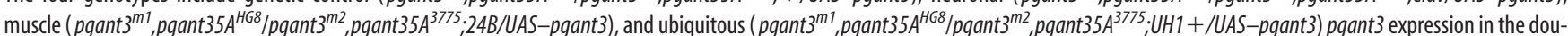
ble mutant. Sample size is $n \geq 10$ animals for all conditions. Representative EJC records $(\boldsymbol{C})$ and mean amplitudes $(\boldsymbol{D})$ for pgant 3 and $p g a n t 35 A$ overexpression conditions. The eight genotypes include pgant3 genetic control (UAS-pgant3/+), neuronal (UAS-pgant3/elav), muscle (UAS-pgant3/24B), and ubiquitous (UAS-pgant3/UH1) pgant3 overexpression, and pgant35A genetic control (UAS-pgant35A/+), neuronal (UAS-pgant35A/elav), muscle (UAS-pgant35A/24B), and ubiquitous (UAS-pgant35A/UH1) pgant35A overexpression. Sample size is $n \geq 10$ animals for each genotype. Statistical differences are calculated using one-way ANOVA with Dunnett's post hoc test: ${ }^{*} p<0.05,{ }^{* *} p<0.01$. N.S., No significance. Error bars indicate SEM. 
synaptic plasticity (Rohrbough et al., 2000). Therefore, we next investigated the multiple phases of activity-dependent plasticity in pgant mutants, including immediate facilitation and maintained augmentation during a tetanic stimulus train $(10 \mathrm{~Hz}$, $60 \mathrm{~s}$ ), and initiation and maintenance of PTP after return to basal stimulation. In this paradigm, EJCs are recorded initially at $0.5 \mathrm{~Hz}$ for $30 \mathrm{~s}$, followed by the tetanic train, and then returned to basal $0.5 \mathrm{~Hz}$ for a total of $5 \mathrm{~min}$ recording (Rohrbough et al., 2000). Figure $5 A$ shows representative traces for control $\left(w^{1118}\right)$, single mutants $\left(\right.$ pgant $3^{m 1} /$ pgant $3^{m 2}$ and pgant $35 A^{H G 8} /$ pgant $35 A^{3775}$ ), and double mutants $\left(\right.$ pgant $3^{m 1}$, pgant $35 A^{H G 8} /$ pgant $3^{m 2}$, pgant $35 A^{3775}$ ).

For quantification, consecutive EJCs are averaged throughout the stimulation phases to display mean amplitudes normalized to the starting level (Fig. 5B). Controls show immediate, rapid facilitation leading to a sixfold augmentation during tetanic stimulation, followed by twofold initial PTP phase, later maintained as an $\sim 50 \%$ elevation for the duration of the recording (Fig. $5 A, B$ ). In contrast, both pgant single mutants show very significantly impaired initial facilitation and blunted fourfold augmentation during tetanic stimulation (Fig. 5B, solid bar labeled C). For example, at $20 \mathrm{~s}$ into the tetanic train, EJC amplitudes show augmentation decreases of $\leq 65 \%$ in pgant $3(p<0.01, n=10)$ and $\leq 55 \%$ in pgant $35 \mathrm{~A}(p<0.01, n=11)$ mutants. In contrast, the double null mutant is clearly less impaired than either single pgant mutant (Fig. $5 A, B$ ). At $20 \mathrm{~s}$ into the tetanic train, the double mutants exhibit a reduced impairment of $\leq 40 \%$ compared with controls ( $p<0.05, n=11$; Fig. $5 C$ ). After this initial facilitation phase, double mutants reach control levels of augmentation, whereas the single pgant mutants remain impaired (Fig. $5 A, B)$, showing a suppressive interaction. After the tetanic train, potentiation in double mutants is indistinguishable from controls, whereas both single mutants (pgant3 and pgant35A) show strong loss of PTP initiation (Fig. 5B, solid bar labeled D). Quantification shows $\geq 50 \%$ decrease in pgant3 $(p<0.001, n=10)$ and $\geq 35 \%$ decrease in pgant $35 A$ ( $p<0.05, n=11)$ single mutants compared with controls but no detectable decrease in the double null mutants (Fig. 5D). Thus, pgant mechanisms regulate activity-dependent facilitation, augmentation, and potentiation.

\section{pgants suppressively regulate integrin signaling}

Synapses sandwich heavily glycosylated transmembrane and extracellular proteins that regulate synaptic function and plasticity (Dani and Broadie, 2012). For example, we have shown previously that O-linked heparan sulfate glycosaminoglycans bidirectionally regulate WNT and BMP trans-synaptic signaling to modulate neurotransmission strength (Dani et al., 2012). To directly visualize changes in synaptic O-GalNAc glycosylation in pgant mutants, we used fluorescently conjugated VVA-TRITC and HPL-Alexa Fluor-488 to label NMJ terminals (Fig. 6). Nondetergent conditions were used throughout to examine only the glycosylation state of the extracellular synaptomatrix (Dani et al., 2012). Representative images show the halo-like VVA (Fig. 6A, top left) and HPL (Fig. 6A, middle left) labeling surrounding the anti-HRP marked synaptic boutons. Compared with controls, O-linked glycan expression is very significantly increased in both pgant3 (31.65 $\pm 5.61 \%, n=9, p<0.01)$ and pgant35A (58.50 \pm $4.39 \%, n=14, p<0.01)$ single mutants, but there is no significant change in the double mutants $(13.54 \pm 5.04 \%, n=6, p>$ 0.05 ) (Fig. $6 B$ ). Similarly, quantified HPL labeling is very significantly elevated in both pgant3 $(33.13 \pm 6.39 \%, n=21, p<0.01)$ and pgant $35 \mathrm{~A}(41.06 \pm 7.83 \%, n=18, p<0.01)$ single mutants, but no significant difference occurs in the double null mutants
(7.49 $\pm 6.85 \%, n=18, p>0.05)$ compared with controls (Fig. 6B). Thus, two independent approaches highlight the suppressive regulation of synaptic O-GalNAc modification by these two pgant genes.

Studies in non-neuronal tissues have shown that pgant mutants misregulate integrin signaling (Zhang et al., 2010). Consistently, we have identified previously presynaptically/postsynaptically localized PS integrin receptors at the Drosophila NMJ, containing multiple different $\alpha$ and $\beta$ subunits (Beumer et al., 1999, 2002; Rohrbough et al., 2000, 2007; Rushton et al., 2009). Therefore, we tested the multiple integrin receptor subunits, including $\alpha \mathrm{PS} 1$ (mew), $\alpha \mathrm{PS} 2$ (if), $\alpha \mathrm{PS} 3$ (scab/volado), $\beta \mathrm{PS}$ (mys; Brower et al., 1984), and $\beta_{\mathrm{v}}$ (Yee and Hynes, 1993). The two $\beta$ subunits show an interesting pgant-specific change, with $\beta P S$ increased in pgant35A (1.39 $\pm 0.08, n=14, p<0.01)$ and $\beta_{\mathrm{v}}$ increased in pgant3 $(1.21 \pm 0.03, n=18, p<0.05)$ single mutants but no significant change of either $\beta$ subunit in the double mu$\operatorname{tant}\left(\beta \mathrm{PS}, 1.17 \pm 0.64, n=10, p>0.05 ; \beta_{\mathrm{v}}, 0.91 \pm 0.06, n=10\right.$, $p>0.05)$ normalized to control. Most of the $\alpha$ receptor subunits show no consistent changes in the pgant mutants, including $\alpha$ PS1 (pgant3, $1.27 \pm 0.07, n=18 ;$ pgant $35 A, 1.10 \pm 0.05, n=18$ ) and $\alpha \mathrm{PS} 3$ (pgant3, $1.05 \pm 0.02, n=8$; pgant $35 A, 1.15 \pm 0.06, n=12$; all $p>0.05$ with respect to control). The sole exception is $\alpha \mathrm{PS} 2$, which sharply decreases in both pgant3 $(39.19 \pm 6.75 \%, n=10$, $p<0.01)$ and pgant $35 A(34.37 \pm 5.69 \%, n=11, p<0.01)$ single mutants but does not change in double mutants compared with controls (Fig. 6B, right).

We next examined a host of characterized integrin ligands for changes in pgant single and double mutants (Zhang and Ten Hagen, 2011), including Tig (Fogerty et al., 1994), laminin $\alpha$ subunits LanA (Inoue and Hayashi, 2007), and Wb (Martin et al., 1999), Tsp (Subramanian et al., 2007), and Ten-m (Levine et al., 1994). Most of these ligands show no consistent changes in pgant single and double mutants compared with control: Tig (pgant3, $1.08 \pm 0.05, n=11 ;$ pgant $35 A, 1.03 \pm 0.05, n=14$; pgant3,pgant $35 A, 1.07 \pm 0.03, n=8$, compared with control, $p>$ 0.05 ), LanA (pgant3, $1.25 \pm 0.07, n=11, p<0.05$; pgant $35 A$, $1.16 \pm 0.07, n=14, p>0.05$; double mutant, $1.00 \pm 0.05, n=8$, $p>0.05), \mathrm{Wb}-\mathrm{N}($ pgant $3,1.00 \pm 0.05, n=10 ;$ pgant $35 \mathrm{~A}, 1.12 \pm$ $0.03, n=11$; double mutant, $1.11 \pm 0.04, n=7$; all $p>0.05)$, and Tsp (pgant3, $1.02 \pm 0.07, n=9 ;$ pgant $35 A, 1.12 \pm 0.06, n=10$; double mutant, $1.13 \pm 0.05, n=8 ; p>0.05$ ), all normalized to control. The sole exception was the RGD domain-containing, transmembrane Ten-m (Levine et al., 1994). Ten-m localizes in a halo-like ring around HRP-labeled synaptic boutons in controls but is consistently reduced in both pgant single mutants (Fig. $6 \mathrm{~A}$, middle). Compared with controls, Ten-m levels are very significantly decreased in pgant3 $(21.88 \pm 3.47 \%, n=15, p<0.01)$ and pgant $35 A(20.84 \pm 3.91 \%, n=16, p<0.01)$ single mutants but show no change in double null mutants (Fig. $6 A$, right). Thus, the two pgant genes suppressively downregulate $\alpha \mathrm{PS} 2$ integrin/ Ten-m ligand at the synapse.

\section{Neuronal and muscle pgants regulate O-glycosylation and integrin signaling}

To determine whether changes in synaptic O-linked glycosylation and trans-synaptic Ten-m/ $\alpha$ PS2 integrin signaling are directed by presynaptic or postsynaptic pgant function, we next tested both pgant 3 and pgant $35 \mathrm{~A}$ rescue in neurons and muscle in their respective null mutant backgrounds (Fig. 7). Representative NMJs showing VVA lectin, $\alpha$ PS2 integrin, and Ten-m ligand labeling are shown for all genotype conditions in Figure $7 \mathrm{~A}$. Both pgant3 neuronal $(1.02 \pm 0.05, n=15)$ and muscle $(1.05 \pm 0.05$, $n=15)$ and pgant $35 A$ neuronal $(1.13 \pm 0.03, n=13)$ and muscle 

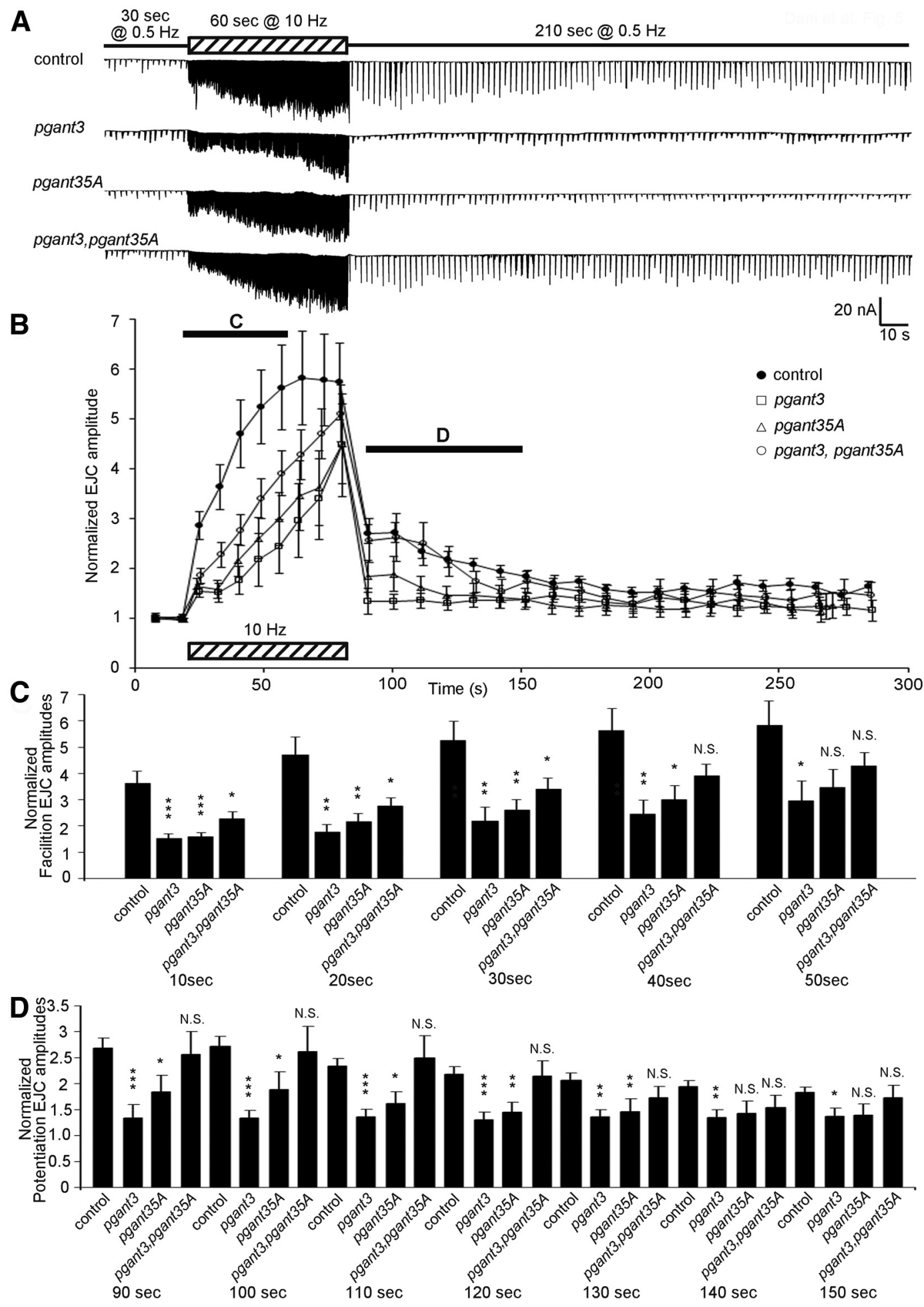

Figure 5. Impaired activity-dependent synaptic plasticity in pgant mutants. $A$, Representative TEVC records from genetic control $\left(w^{1118}\right.$; top), pgant3 $\left(\right.$ pgant $\left.3^{m 1} / p g a n t 3^{m 2}\right)$ and $p g a n t 35 A$

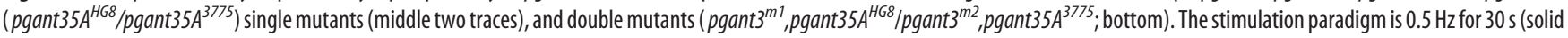
bar), $10 \mathrm{~Hz}$ for $60 \mathrm{~s}$ (hatched bar), and then a return to $0.5 \mathrm{~Hz}$ (solid bar) for $5 \mathrm{~min}$ total recording time. Calibration: $10 \mathrm{~s}, 20 \mathrm{nA}$. B, Mean EJC amplitudes over time normalized to initial mean EJC amplitude for control (filled circle), pgant3 (open square) and pgant35A (open triangle) single mutants, and double mutant (open circle). Bars labeled C and D are expanded below, showing normalized EJC amplitudes during tetanic stimulation $(\boldsymbol{C}$ ) and early posttetanic stimulation periods (D). Sample sizes are $n \geq 10$ independent NMJs for each of the four genotypes. Statistical differences are calculated using one-way ANOVA with Dunnett's post hoc test: ${ }^{*} p<0.05,{ }^{* *} p<0.01,{ }^{* * *} p<0.001$. N.S., No significance. Error bars indicate SEM.

$(1.01 \pm 0.03, n=22)$ expression restored VVA lectin labeling to control levels $(1.00 \pm 0.03, n=18$; Fig. $7 B)$. Similarly, $\alpha$ PS2 integrin abundance is also rescued with pgant 3 neuronal $(0.94 \pm$ $0.05, n=19)$ or muscle $(0.99 \pm 0.06, n=20)$ expression and
pgant35A neuronal $(0.90 \pm 0.04, n=21)$ or muscle $(0.86 \pm 0.04$, $n=25)$ expression compared with control (1.00 $\pm 0.03, n=20$; Fig. $7 B)$. Interestingly, only neuronal pgant3 $(0.99 \pm 0.04, n=$ $10)$ and pgant $35 A(0.97 \pm 0.04, n=8)$ expression could restore 
A
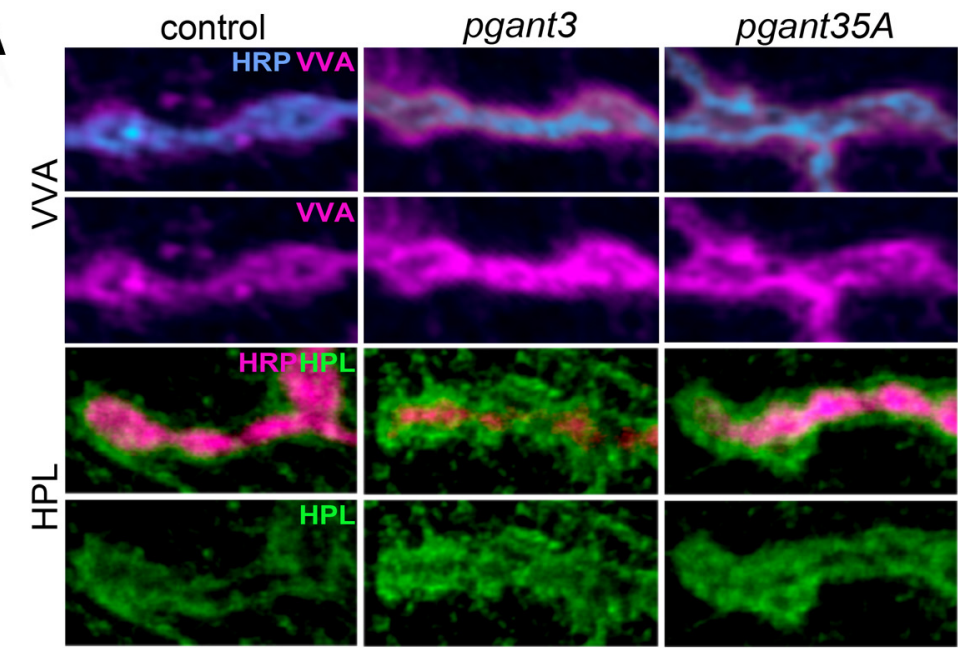

pgant3,pgant35A
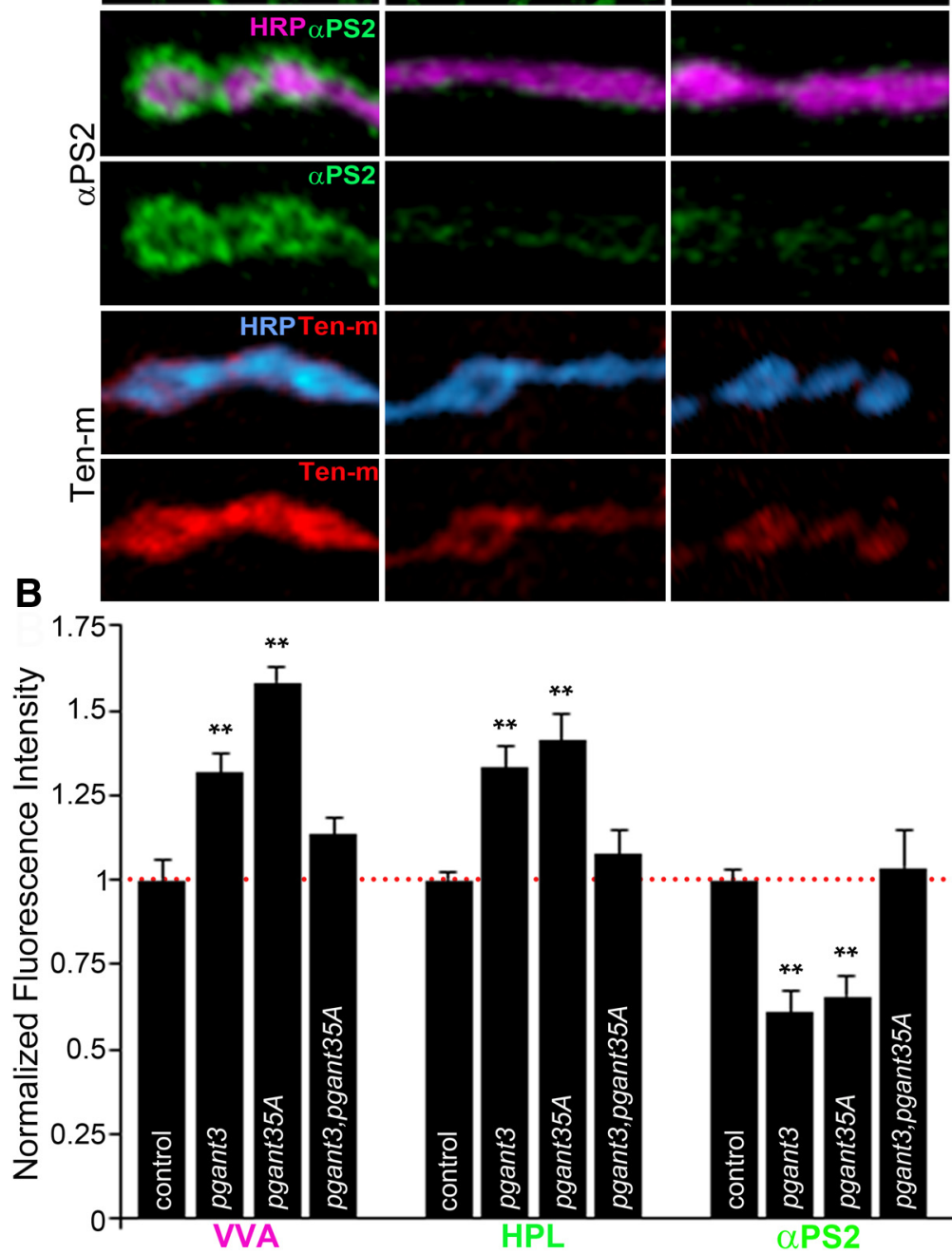

aPS2
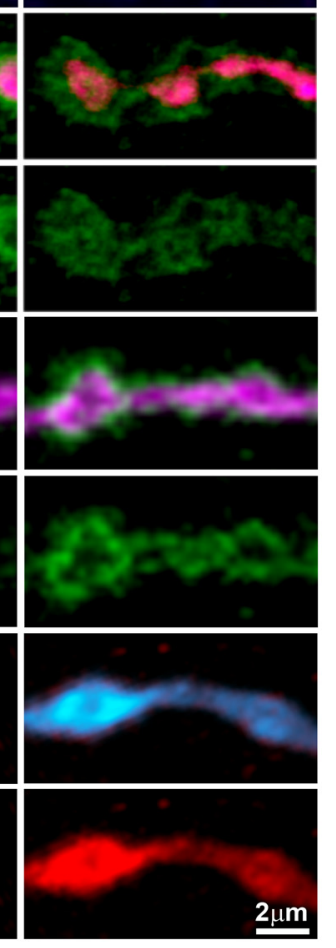

Figure 6. Synaptomatrix 0-glycan and integrin signaling defects in pgant mutants. $A$, NMJ synaptic boutons from control $\left(w^{1118}\right.$; left column), pgant3 $\left(\right.$ pgant $\left.3^{m 1} / p g a n t 3^{m 2}\right)$ and pgant35A

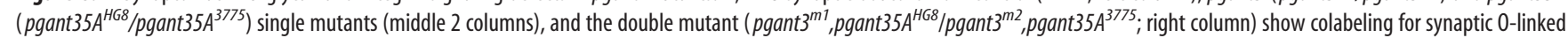
glycosylation markers (VVA and HPL), integrin receptor ( $\alpha$ PS2), and transmembrane integrin ligand (Ten-m) relative to the presynaptic marker anti-HRP. Scale bar, $2 \mu \mathrm{m} . \boldsymbol{B}$, Histograms show fluorescence intensities for all four labels (VVA, HPL, $\alpha$ PS2, and Ten-m) normalized to each genetic control (dotted red line). Sample size is $n \geq 10$ independent NMJs for each label for all four genotypes. Statistical differences calculated using one-way ANOVA with Dunnett's post hoc test: ${ }^{* *} p<0.01$. Error bars indicate SEM.

synaptic Ten-m levels to control levels $(1.00 \pm 0.03, n=21)$, whereas muscle pgant3 $(0.88 \pm 0.04, n=17, p<0.05)$ and pgant35A $(0.82 \pm 0.03, n=14, p<0.01)$ remained significantly decreased normalized to control (Fig. $7 B$, right).
Thus, both presynaptic and postsynaptic pgant 3 and pgant $35 \mathrm{~A}$ are sufficient to properly regulate synaptic O-linked glycosylation and integrin levels, but regulation of the Ten-m ligand requires pgant function in the presynaptic neuron. 
A
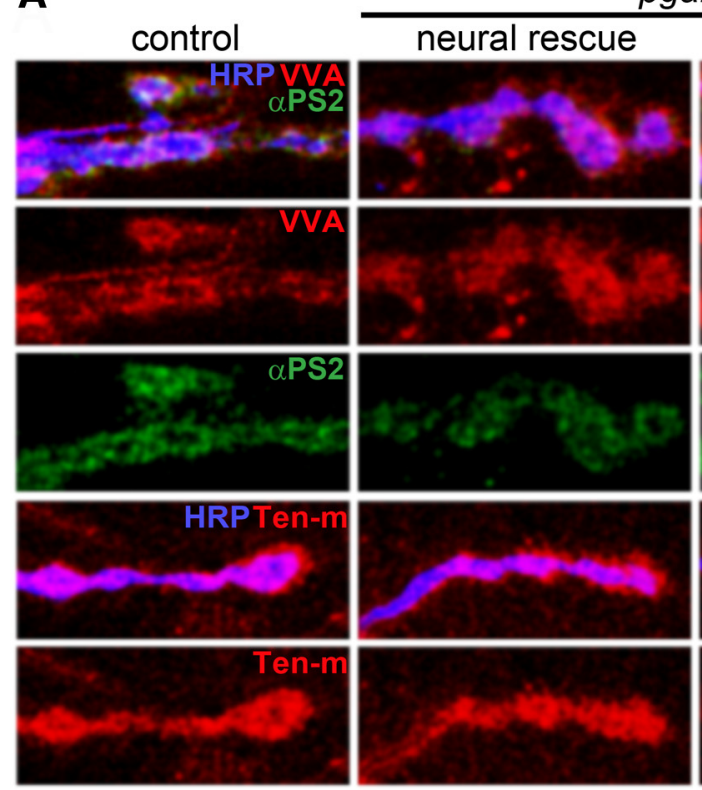

pgant3
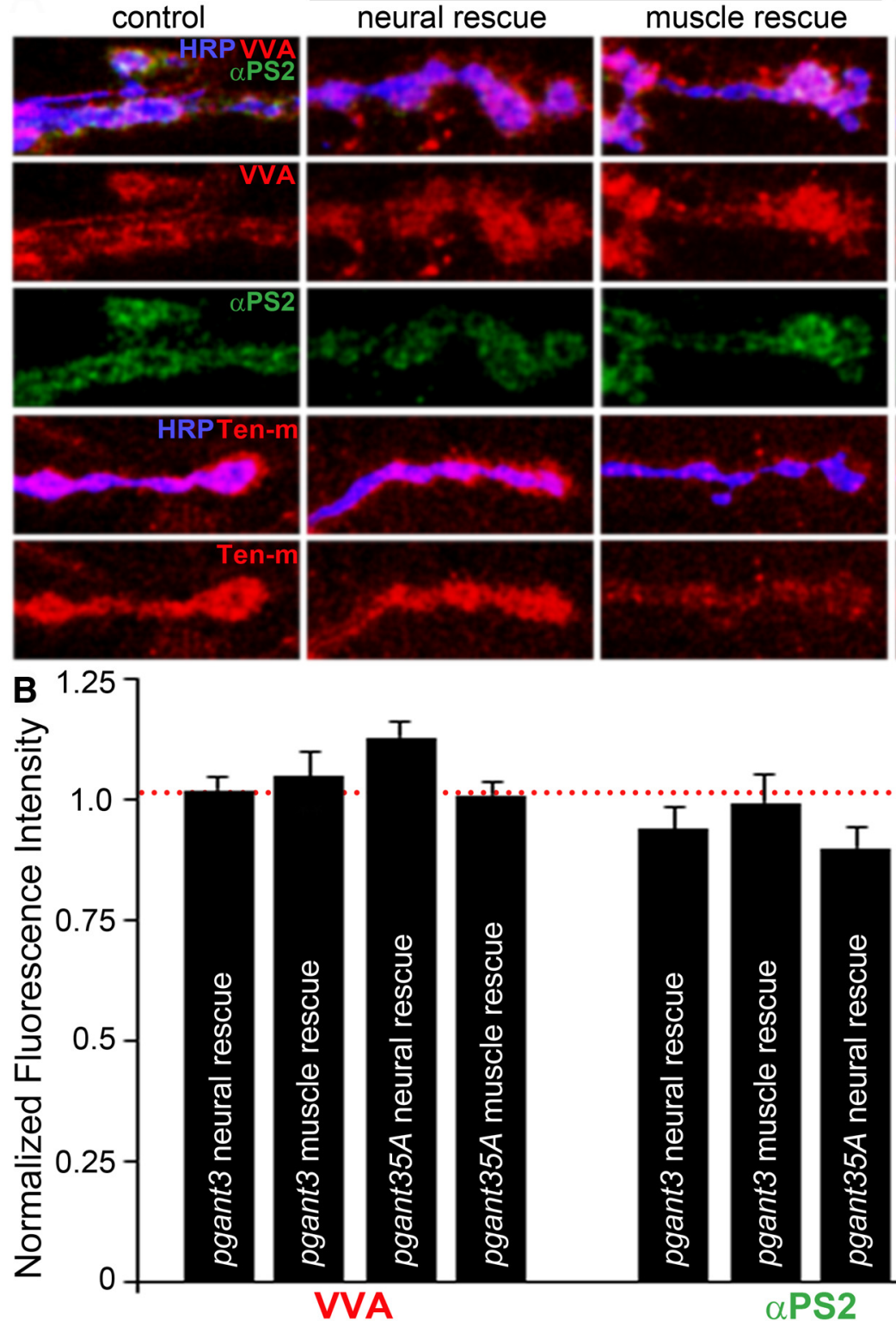

pgant35A

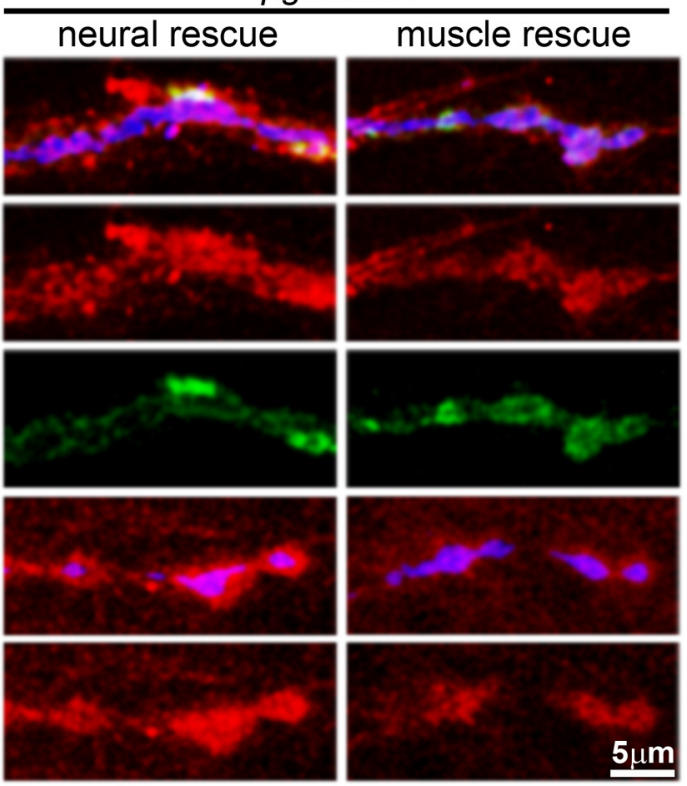

Figure 7. Presynaptic/postsynaptic pgant $3 / 35$ A regulate 0 -GalNAc and integrin signaling. $A$, NMJ synaptic boutons colabeled for synaptic 0 -linked glycosylation marker (VVA), integrin receptor ( $\alpha$ PS2; top 3 panels), and Ten-m relative to the presynaptic marker anti-HRP (bottom 2 panels), with split channels shown for clarity. The five genotypes shown include genetic control ( $w^{1118}$ ),

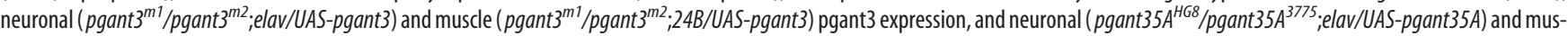
cle ( pgant35A ${ }^{H G 8} /$ pgant35A $A^{3775} ; 24 B / U A S-p g a n t 35 A$ ) pgant35A expression conditions. Scale bar, $2 \mu \mathrm{m}$. B, Histograms showing the relative fluorescence intensities for all three labels (VVA, $\alpha$ PS2, and Ten-m) normalized to each genetic control (dotted red line). Sample size is $n \geq 8$ independent NMJs for each label for all five genotypes. Statistical differences calculated using one-way ANOVA with Dunnett's post hoc test: ${ }^{*} p<0.05,{ }^{* *} p<0.01$. Error bars indicate SEM.

pgants regulate activity-dependent integrin signaling at the synapse

With striking activity-dependent effects on synaptic plasticity in pgant mutants (Fig. 5), we next queried activity-dependent changes in integrin signaling (Fig. 8). Channelrhodopsinmediated optogenetic stimulation was used to drive presynaptic activity, followed by confocal microscopy examination for molecular changes at the NMJ synapse. The neuronal driver (elav-gal4; Lin and Goodman, 1994) was used to target UASChIEF-tdTomato (Wang et al., 2011) in genetic control, single mutants, and double mutants. Channelrhodopsin targeting was confirmed by visualizing tdTomato expression (Fig. $8 \mathrm{~A}$ ) and eliciting EJPs with $5 \mathrm{~Hz}$ blue light $(\lambda=460 \mathrm{~nm}, 60 \mathrm{~ms}$ duration) stimulation (Fig. 8B). Guided by the plasticity stimulation paradigm (Fig. 5), preparations were illuminated with $60 \mathrm{~ms}$ light pulses at $10 \mathrm{~Hz}$ for $60 \mathrm{~s}$ and then immediately fixed for imaging (Fig. 8C). VVA-TRITC O-GalNac labeling did not detectably change in unstimulated controls compared with optogenetically stimulated preparations (pgant3, $1.09 \pm 0.04$; pgant35A, $1.09 \pm$ 0.03 ; double mutant, $0.96 \pm 0.04$ as normalized to controls; $n \geq$ 13; all $p>0.05)$. Similarly, we observe no change in levels of integrin ligand Ten-m (pgant3, $1.00 \pm 0.04$; pgant35A, $1.02 \pm$ 1.04; double mutant, $1.14 \pm 0.05 ; n \geq 17$; all $p>0.05$ ) or integrin receptor $\alpha$ PS2 levels (pgant3, $0.94 \pm 0.04$; pgant $35 A, 0.88 \pm 0.04$; double mutant, $0.84 \pm 0.07$ as normalized to controls; $n \geq 8$; all $p>0.05)$. Therefore, we investigated integrin downstream sig- 
A

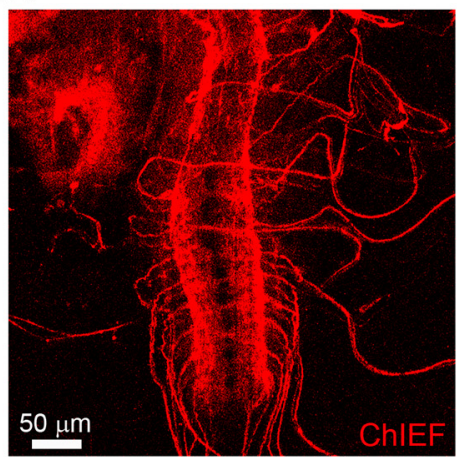

C

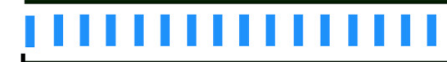

$30 \mathrm{sec}$
B

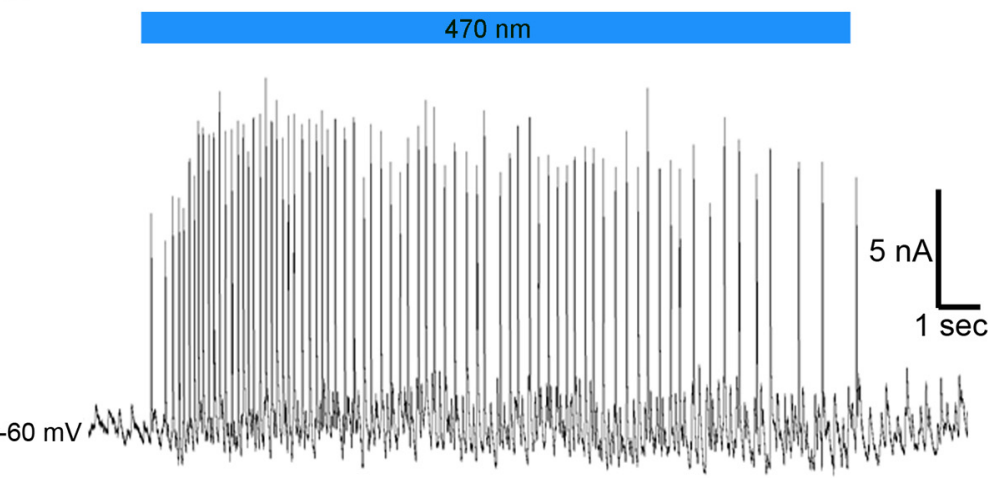

Optogenetic stimulation

D

UNSTIMULATED
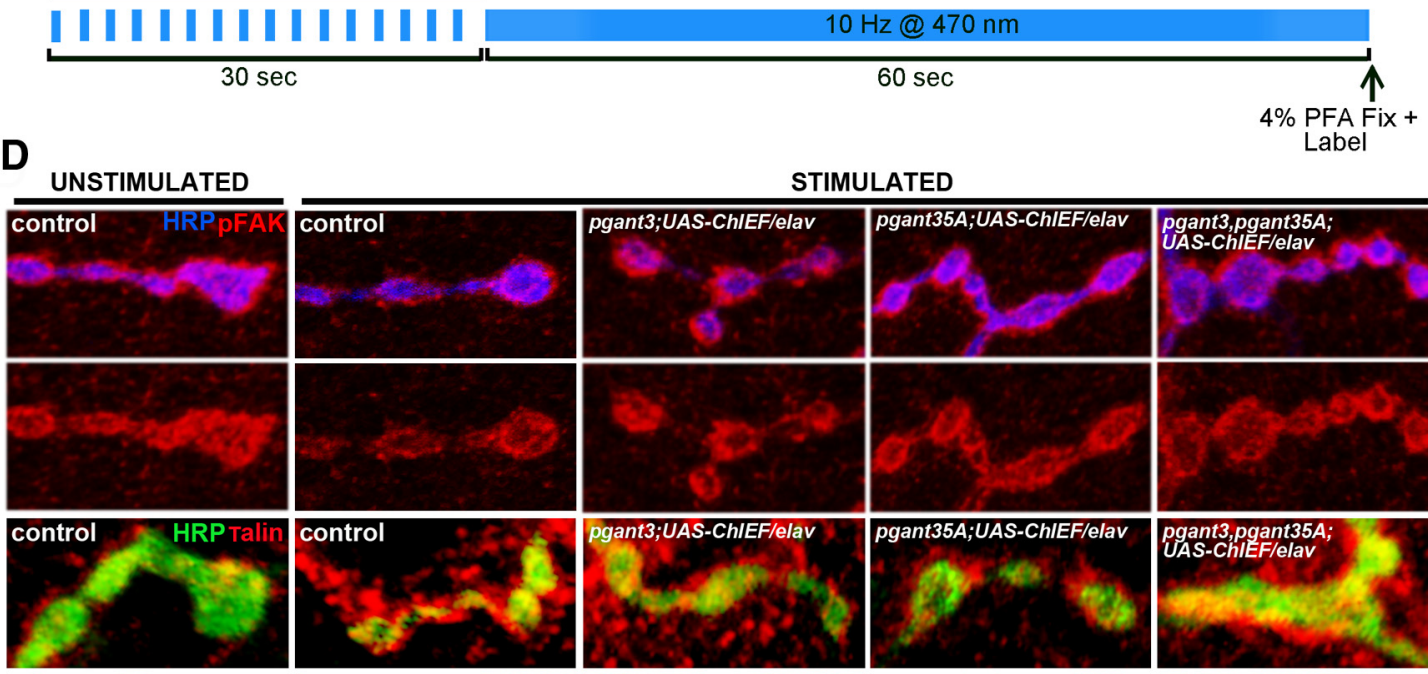

STIMULATED
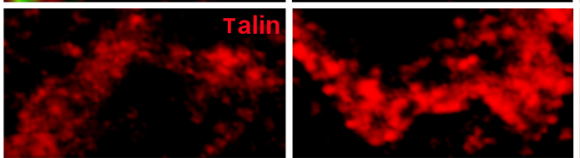

E $\quad 1.25$

pFAK

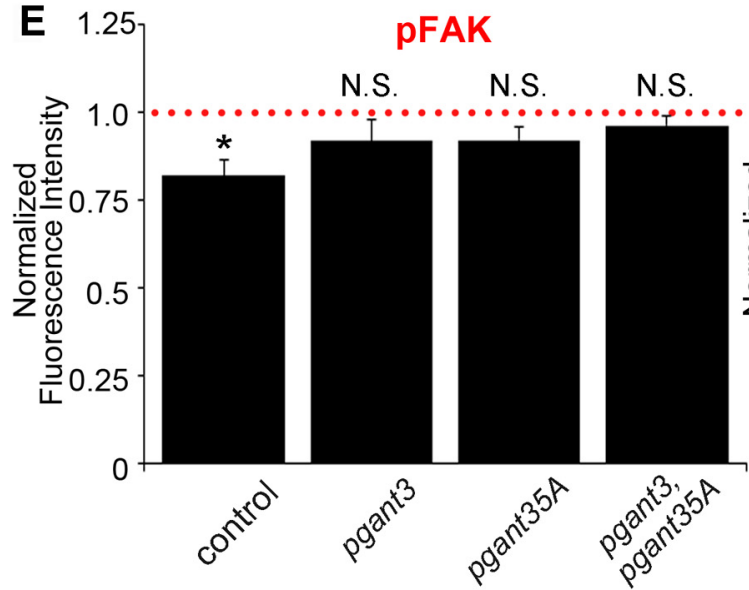

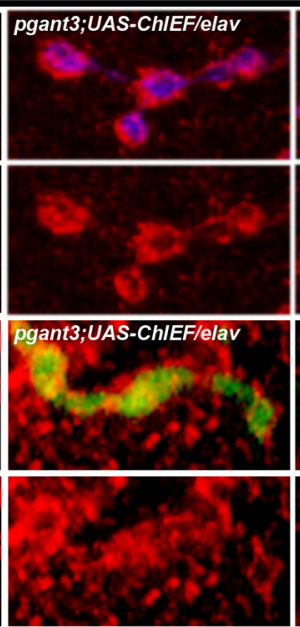

1.5
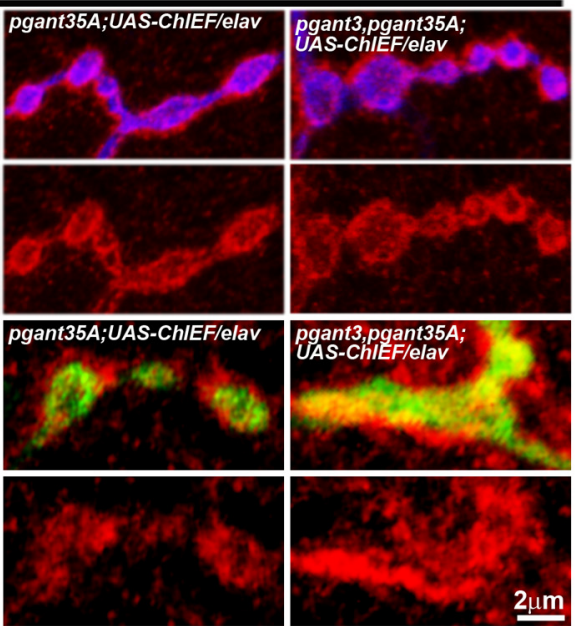

pgant3,pgant35A;
UAS-ChlEFlelav
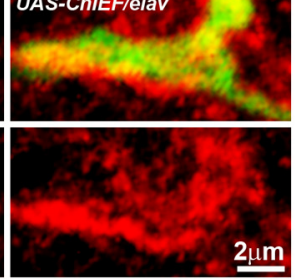

$2 \mu \mathrm{m}$

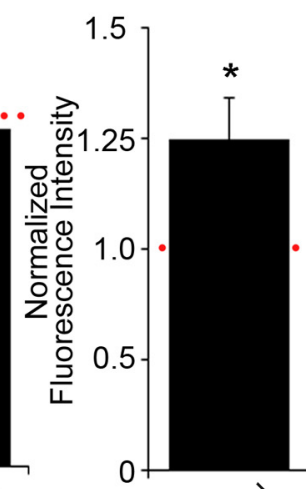

Talin

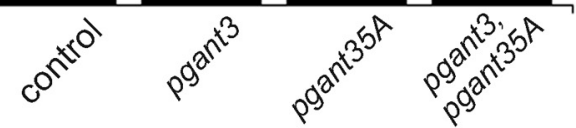

Figure 8. Activity-dependent integrin signaling changes in pgant mutants. $\boldsymbol{A}$, Representative image of neurally targeted (elav-ga/4) channelrhodopsin expression (ChIEF-tdTomato) in third-instar ventral nerve cord. The native fluorescence of the tagged light-activated channel (red) is shown without amplification. Scale bar, $50 \mu \mathrm{m} . \boldsymbol{B}$, Representative EJP trace from the muscle 6 NMJ induced by blue light $(470 \mathrm{~nm}$ ) stimulation of ChlEF-expressing neurons. C, Schematic of the optogenetic stimulation paradigm used to assay changes in integrin signaling. Basal stimulation at $0.5 \mathrm{~Hz}$ for $30 \mathrm{~s}$ to establish baseline, followed by $10 \mathrm{~Hz}$ blue light pulses for $60 \mathrm{~s}$ to induce activity-dependent changes. Samples were then immediately fixed and labeled for analyses. $\mathbf{D}$, Representative images of pFAK (top) and Talin (bottom) labeling, with HRP colabeling, showing split channels to compare unstimulated control (left) and light-stimulated (10 Hz, $60 \mathrm{~s}$ ) synaptic

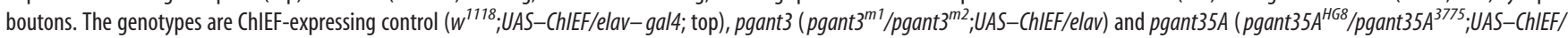
elav-gal4) single mutants (middle 2 rows), and the double mutant (pgant $3^{m 1}$, pgant $35 A^{H G 8} /$ pgant $3^{m 2}$,pgant $35 A^{3775} ;$,UAS-ChIEF/elav- gal4 (far right). E, Histograms show relative fluorescence intensities of pFAK (left) and Talin (right) normalized to genetic control for all four genotypes expressing ChIEF. Sample size is $n \geq 8$ independent NMJs for each label and condition for all genotypes. Statistics calculated using one-way ANOVA with Dunnett's post hoc test: ${ }^{*} p<0.05$. Error bars indicate SEM. 
naling by assaying Talin and pFAK abundance (Devenport et al., 2007; Tsai et al., 2012a).

To determine whether activity-dependent integrin signal transduction is affected, we investigated channelrhodopsindependent changes in Talin recruitment and downstream pFAK production (Fig. $8 D$ ). Interestingly, when compared with respective unstimulated genotype controls, optogenetic stimulation drives a striking increase in Talin levels in both control (49.41 \pm $18.96 \%, n=8, p<0.05)$ and pgant double mutants $(80.1 \pm$ $7.93 \%, n=13, p<0.05)$ compared with unstimulated conditions, whereas neither pgant3 nor pgant $35 \mathrm{~A}$ single mutants showed any significant activity-dependent change in Talin recruitment to the synapse (Fig. $8 D$, bottom row, $E$, right). Moreover, we find an activity-dependent decrease pFAK levels in stimulated controls (UAS-ChIEF/elav, $17.85 \pm 4.23 \%, n=49$, $p<0.05)$, with no change in stimulated pgant3 (pgant $3^{\mathrm{ml}} /$ pgant $^{m 2}$;UAS-ChIEF/elav, $\left.8.15 \pm 6.28 \%, n=43, p>0.05\right)$, pgant35A (pgant35A $A^{H G 8} /$ pgant $35 A^{3775}$;UAS-ChIEF/elav, $7.76 \pm$ $3.94 \%, n=61, p>0.05)$, and double mutant pgant3,pgant $35 \mathrm{~A}$ ( pgant $^{m 1}{ }^{m 1}$,pgant $35 A^{H G 8} /$ pgant $^{m 2}{ }^{\text {}}$,pgant $35 A^{3775}$;UAS-ChIEF/ elav, $3.61 \pm 2.61 \%, n=27, p>0.05$ ) conditions (Fig. $8 D$, top row, $E$, left). We conclude that both integrin recruitment of Talin and downstream production of pFAK is activity dependent and under pgant dependent suppressive regulation.

\section{pgants regulate activity-dependent PSP size}

Misregulated integrin signaling leads to intercellular de-adhesion and subsequent wing blistering in pgant mutant wing discs (Zhang et al., 2010). Moreover, mutants in trans-synaptic WNT/ BMP and HSPG extracellular pathways manifest enlarged PSPs at the NMJ (Packard et al., 2002; Kamimura et al., 2013). Because we have shown that pgant mutants suppressively regulate basal and activity-dependent integrin signaling and PSP expansion, we next examined optogenetic activity-dependent synaptic ultrastructural changes, with a particular focus on the PSP. In the above channelrhodopsin-expressing mutants and controls, we adopted the same light stimulation paradigm, followed by fixation and transmission electron microscope (TEM) examination of synapse ultrastructure (Fig. 9).

In optogenetically stimulated synaptic terminals, there is an obvious decrease in SV density in all four genotypes compared with unstimulated controls (Fig. 9A). At $<250 \mathrm{~nm}$ away from the active zone, quantification of SV number shows an $\sim 30 \%$ decrease in controls and a similar $\sim 30 \%$ decrease in stimulated pgant 3 mutants $(n=13, p<0.001$ compared with unstimulated condition; Fig. 9B). Both the pgant35A single mutant $(n=19)$ and the double null mutant $(n=16)$ behave similarly. Furthermore, there are no significant differences in suppressive regulation under basal conditions (Fig. 2) or unstimulated UAS-ChIEF carrying lines (Fig. 9) i.e., single mutants ( pgant $3^{m 1} /$ pgant $^{m 2}$, $15.22 \pm 0.99$ vesicles; pgant $35 A^{H G 8} /$ pgant $35 A^{3775}, 15.54 \pm 0.78$; $p<0.05)$ are elevated compared with the control $\left(w^{1118}, 10.5 \pm\right.$ 0.91 ) and the double mutant (pgant $3^{m 1}$,pgant $35 A^{H G 8}$ / pgant $3^{m 2}$,pgant $\left.35 A^{37758}, 9.4 \pm 0.67\right)$. Similarly, in unstimulated single mutants carrying the channelrhodopsin transgene, SVs are elevated $\left(\right.$ pgant $^{m 1} /$ pgant $^{m 2}$; UAS-ChIEF/elav, $9.53 \pm 0.63, p<$ 0.01; pgant $35 A^{\text {HG8 }} /$ pgant $35 A^{3775}$;UAS-ChIEF/elav, $8.95 \pm 0.49$, $p<0.05)$ with respect to control (UAS-ChIEF/elav, $7.13 \pm 0.57$ ) and double mutant (pgant ${ }^{m 1}$, pgant $35 A^{H G 8} /$ pgant $^{m 2}$,pgant $35 A^{3775}$; UAS-ChIEF/elav, $7.68 \pm 0.39$ ). Thus, activity drives SV cycling in all four genotypes comparably (Fig. 9B).

In contrast, optogenetically stimulated control NMJ synapses show an activity-dependent increase in PSP depth that does not occur in either pgant 3 and pgant $35 \mathrm{~A}$ single mutant, although the double mutant is indistinguishable from control (Fig. 9A, dotted lines). Quantification of these differences reveal an activitydependent PSP depth increase of $>50 \%$ in control $(p<0.05$ compared with unstimulated condition) and $\sim 35 \%$ increase in the double null mutants $(n=14, p<0.05)$ but no significant change in either single pgant mutant (Fig. $9 C$ ). Furthermore, comparing basal genotypes with unstimulated ChIEF carrying controls and mutants show no significant difference in PSP depth, for controls ( $w^{1118}, 120.47 \pm 7.46 \mathrm{~nm}, n=12$ vs unstimulated control, $147.41 \pm 15.69 \mathrm{~nm}, n=14, p>0.05)$, single mutants (pgant3, $272.36 \pm 45.83 \mathrm{~nm}, n=13$ vs unstimulated pgant $3,253.26 \pm 37.70 \mathrm{~nm}, n=14, p>0.05 ;$ pgant $35 A, 246.84 \pm$ $46.63 \mathrm{~nm}, n=12$ vs unstimulated pgant $35 \mathrm{~A}, 232.97 \pm 24.88 \mathrm{~nm}$, $n=15, p>0.05)$ and the double mutant (pgant3,pgant35A, $182.13 \pm 23.76 \mathrm{~nm}, n=14$ vs. unstimulated pgant3, pgant $35 \mathrm{~A}$, $227.31 \pm 18.56 \mathrm{~nm}, n=25, p>0.05)$. Thus, presynaptic vesicle number decreases in all genotypes with acute optogenetic stimulation, but pgants suppressively regulate activity-dependent PSP expansion, consistent with the dysregulated integrin-mediated signaling.

\section{Integrin inhibition blocks activity-dependent synaptic plasticity in pgant mutants}

We have shown previously that blocking integrin signaling with RGD peptides interferes with synaptic plasticity at the Drosophila NMJ, comparably with integrin mutations (Bahr et al., 1997; Rohrbough et al., 2000). Furthermore, the Ten-m integrin ligand that is found to be suppressively regulated by pgants contains an RGD sequence. Hence, as a direct test of integrin signaling requirements in pgant-dependent facilitation, augmentation, and potentiation phases of tetanic stimulus train-induced synaptic plasticity, we used RGD integrin inhibitory peptides and scrambled RAD controls in the genetic background control, pgant single mutants, and the double mutant (Rohrbough et al., 2000). Using our established protocols for peptide incubation (Rohrbough et al., 2000), we recorded EJCs using the same stimulation paradigm used above (Fig. 5). Recordings were normalized to the mean basal EJC amplitude in sham/RGD/RAD-treated controls (Fig. 10A), pgant3 (Fig. 10B) and pgant35A (Fig. 10C) single mutants, and pgant3,pgant $35 \mathrm{~A}$ (Fig. 10D) double mutants. Consecutive EJCs were averaged during the 0.5 and $10 \mathrm{~Hz}$ stimulation phases, respectively, for data presentation and quantification.

In RGD-treated compared with RAD-treated control $\left(w^{1118}\right)$ synapses, a $>50 \%$ elevation occurs in synaptic augmentation during the tetanic stimulus train, and $>30 \%$ increase occurs in PTP after stimulation (Fig. 10A). In striking contrast, pgant single and double mutants show a synergistic interaction with integrin blockade to exhibit a loss of both phases of activity-dependent plasticity (Fig. 10B-D, left). Quantification of EJC amplitudes during the tetanic phase shows a significant increase in RGDtreated compared with RAD-treated control synapses $(p<0.05$, $n \geq 9$; Fig. 10A, right). However, EJC amplitudes actually decrease $\sim 60 \%$ in pgant3 ( $p<0.05, n \geq 9$; Fig. $10 B$, right), pgant35A ( $p<0.05, n \geq 6$; Fig. $10 C$, right), and pgant3,pgant $35 \mathrm{~A}$ $(p<0.05, n \geq 4$; Fig. 10D) after RGD treatment. During PTP phases, RGD treatment again causes a highly significant EJC amplitude increase compared with RAD-treated controls $(p<0.05$, $n \geq 9$; Fig. 10A, right). Remarkably, RGD treatment instead causes $>50 \%$ decreases in pgant $3(p<0.05, n \geq 9$; Fig. 10B), pgant35A ( $p<0.05, n \geq 6$; Fig. $10 C)$, and pgant3,pgant $35 \mathrm{~A} \mathrm{mu-}$ tants ( $p<0.05, n \geq 4$; Fig. 10D) compared with RAD-treated synapses. Importantly, there are no significant differences be- 
A
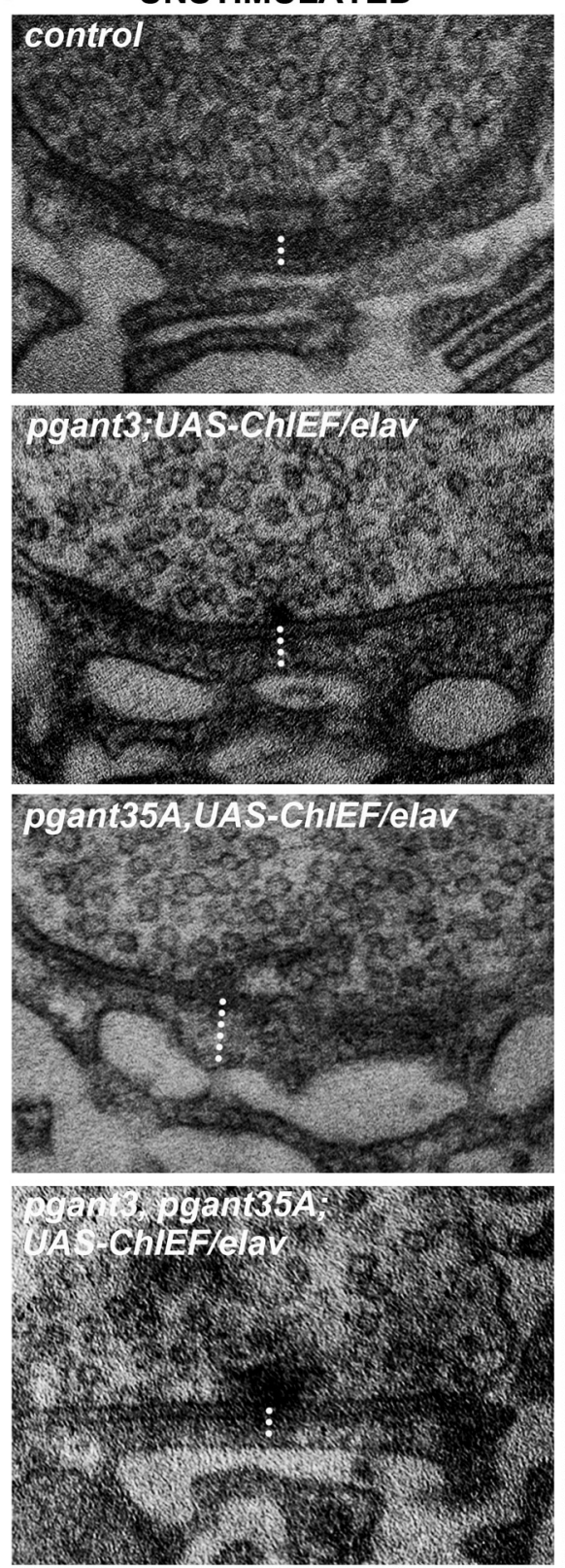

STIMULATED

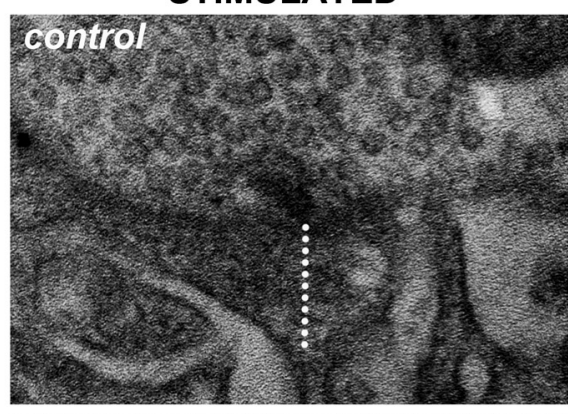

P.
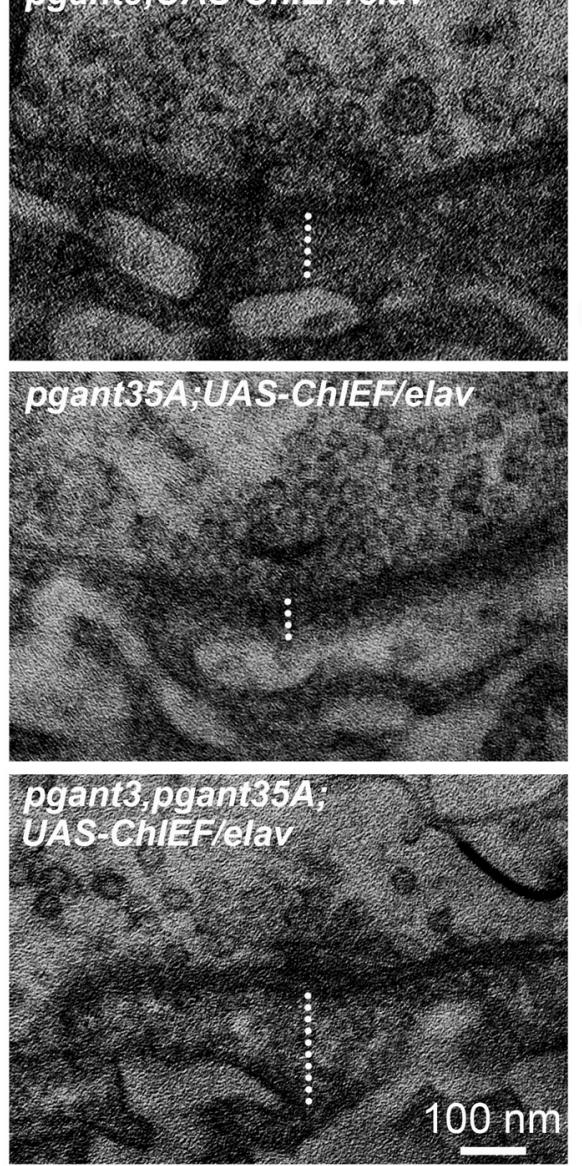

B
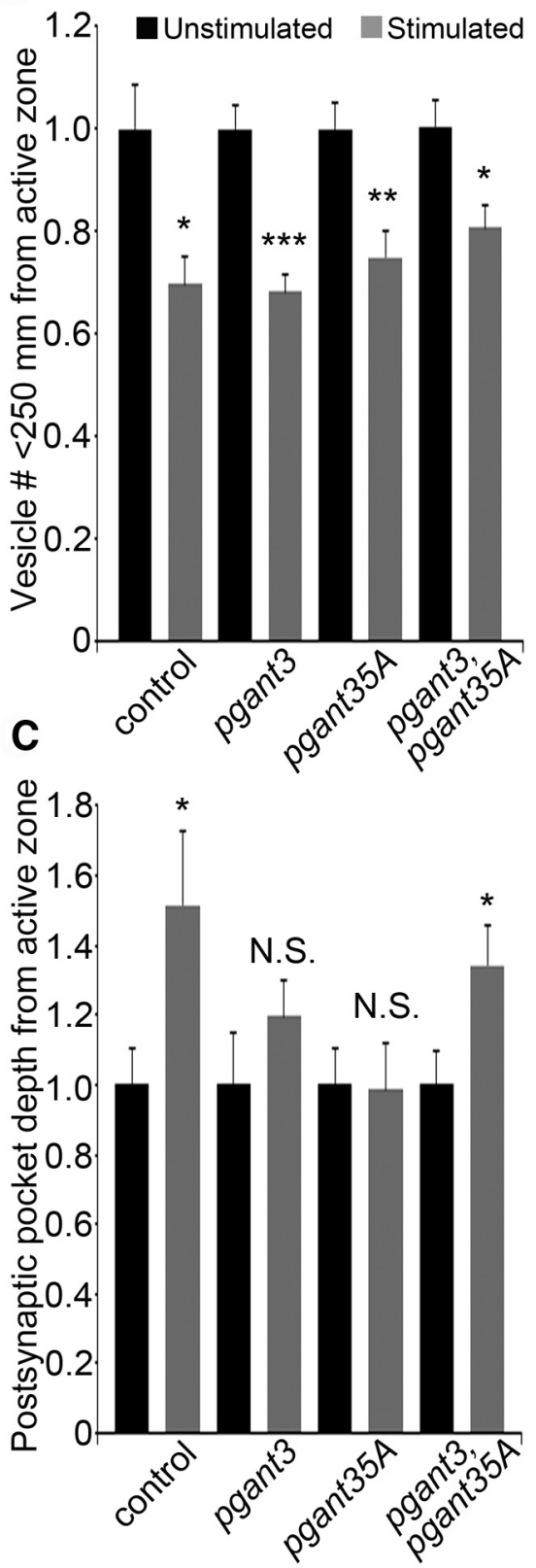

Figure 9. Activity-dependent changes in synapse ultrastructure in pgant mutants. A, Representative active zone synapses of control ( $w^{1118}$;UAS-ChIEF/elav- gal4; top), pgant3 ( pgant $3^{m 1 / m 2}$;

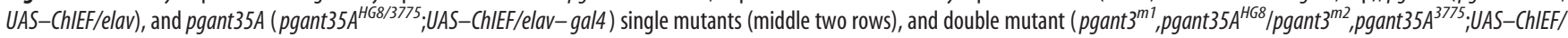
elav-gal4, bottom) when comparing the unstimulated (left column) and light-stimulated ( $10 \mathrm{~Hz}, 60 \mathrm{~s}$; right column) conditions. Dotted white line indicates the PSP depth for each condition and genotype. Scale bar, $100 \mathrm{~nm}$. B, C, Histograms showing quantification of activity-dependent changes for normalized SV number $(\boldsymbol{B})$ and PSP depth $(\boldsymbol{C})$ for all four genotypes under unstimulated (black bars) and stimulated (gray bars) conditions. Sample size is $n \geq 15$ independent boutons for each genotype and condition. Statistical differences are calculated using one-way ANOVA with Dunnett's post hoc test: ${ }^{*} p<0.05,{ }^{* *} p<0.01,{ }^{* * *} p<0.001$. N.S., No significance. Error bars indicate SEM.

tween RAD-treated synapses and sham controls (Fig. 10A-D). We conclude that integrin signaling blockade coupled to the loss of pgant function causes a complete loss of activity-dependent facilitation, augmentation, and potentiation, consistent with a requirement of pgant activity in integrin-mediated functional synaptic plasticity.

\section{Discussion}

Across species, glycans are increasingly being recognized as key regulators of synaptic function and plasticity (Dani and Broadie, 2012; Scott and Panin, 2014). Classically, $\operatorname{Gal}(\beta 1,4)$ GlcNAc, $\operatorname{Gal}(\beta 1,3)$ GalNAc, CT carbohydrate antigen, heparin, heparan sulfate, and sialic acid are all known to modulate the trans-synaptic agrin signal mediating postsynaptic acetylcholine receptor stabilization at mammalian NMJs (Wallace, 1990; Parkhomovskiy et al., 2000). Similarly, the Drosophila Mtg glycan-binding lectin regulates the stabilization/organization of postsynaptic glutamate receptors and establishes the extracellular matrix-integrin interface at the NMJ (Rohrbough et al., 2007; Rushton et al., 2009). Other Drosophila glycan regulating genes, including sialyltransferase, sialic acid transporter Fuseless, and Mgat1, also modulate ion channels, presynaptic/postsynaptic organization, and neurotransmission strength at the NMJ (Long et al., 2008; Repnikova 

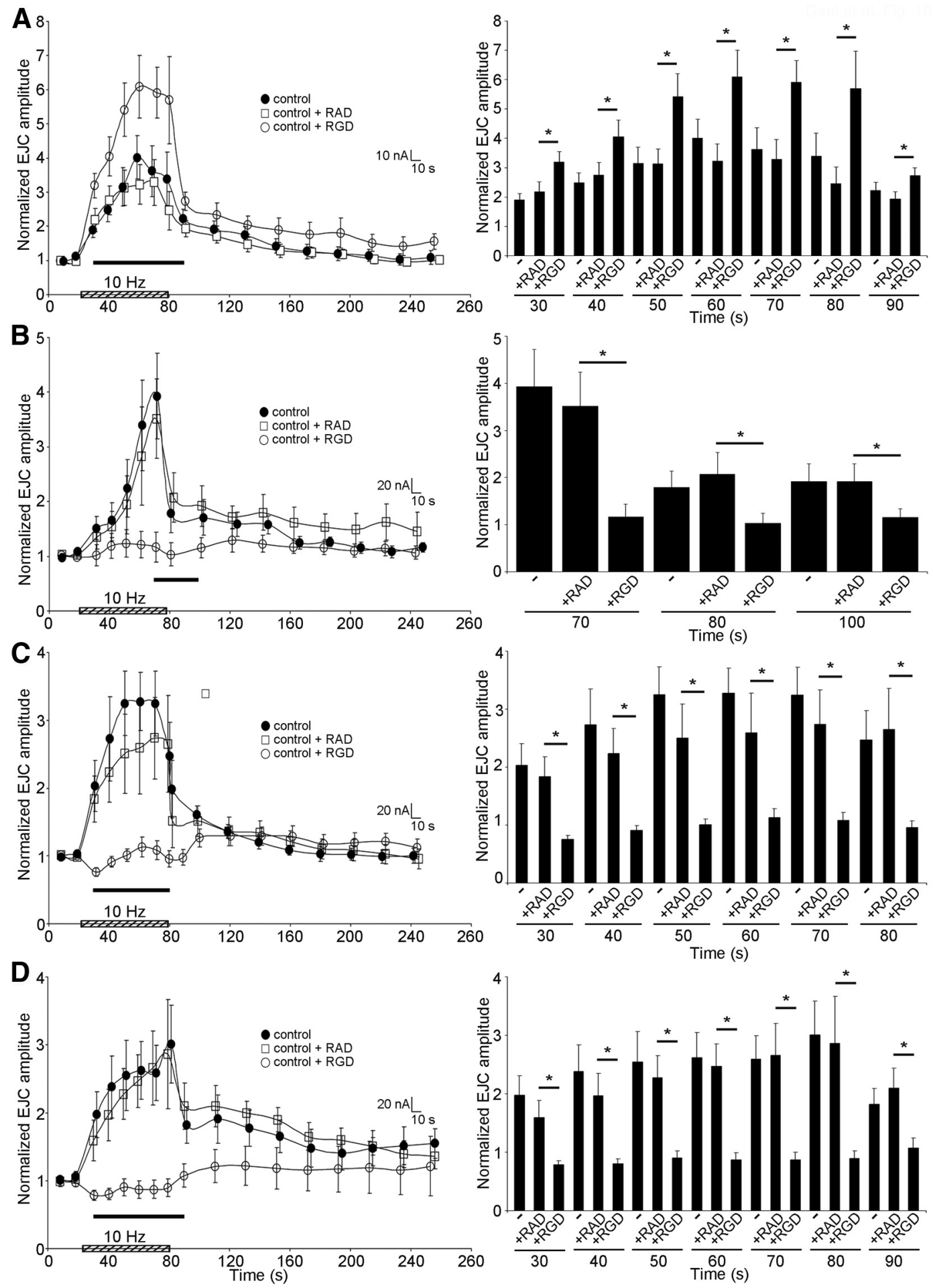

Figure 10. Integrin inhibition blocks all synaptic plasticity in pgant mutants. TEVC recordings from sham-treated, RAD control, and RGD integrin-blocking peptide applications in genetic control

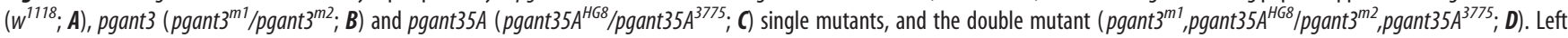
column, The stimulation paradigm is indicated on the $x$-axis with EJC amplitudes normalized to the basal EJC amplitude in each condition for sham control (filled circle), RAD control (open square), and integrin-blocking RGD (open circle) peptide applications. Right column, Histograms show normalized EJC amplitudes for sham/RAD/RGD peptide treatments for the indicated time periods. Samples size is $n \geq 5$ independent NMJs for each genotype and treatment condition ( $>60$ recordings total). Statistical differences calculated using one-way ANOVA with Dunnett's post hoc test: ${ }^{*} p<0.05$. Error bars indicate SEM.

et al., 2010). Our RNAi glycogene screen recently identified a pair of genes (hs6st and sulf1) that regulate HSPG sulfation state to modulate the bidirectional trans-synaptic WNT/BMP signaling driving presynaptic/postsynaptic assembly and synapse function (Dani et al., 2012; Parkinson et al., 2013). Another gene pair, pgant 3 and pgant $35 \mathrm{~A}$, catalyzing early steps of mucin O-glycan (GalNAc $\alpha 1-\mathrm{O}-\mathrm{S} / \mathrm{T}$ ) posttranslational modification as $\mathrm{N}$-acetylgalactosaminyl transferases (Schwientek et al., 2002; Ten Hagen et al., 2003b), was identified to have neurotransmission effects in the same screen. 
In Drosophila, pgant3 is characterized to regulate integrinligand secretion and intercellular adhesion and pgant $35 \mathrm{~A}$ for appropriate intercellular septate junction formation (Tian and Ten Hagen, 2007; Zhang et al., 2008). Microarray analyses have identified pgant 3 and pgant $35 \mathrm{~A}$ transcripts in the developing nervous system and musculature (Tian and Ten Hagen, 2006; Chintapalli et al., 2007), and our lectin analyses show NMJ O-GalNAc modifications dependent on both pgant 3 and pgant $35 \mathrm{~A}$. Null mutants display increased presynaptic active zone $b r p$ (ELKS/CAST) and postsynaptic glutamate receptor bad reception (GluRIID) assembly (Featherstone et al., 2005; Wagh et al., 2006) and elevated evoked neurotransmission strength, and genetic rescue experiments show pgant 3 and pgant $35 \mathrm{~A}$ function in both neurons and muscle. All synaptic defects occurring in single pgant nulls are absent in double mutants, which are essentially indistinguishable from controls. Similar observations have been described as "reciprocal suppression" in the context of physically interacting proteins, respectively (Honts et al., 1994). However, because the basis of the pgant3/pgant35A interaction is as yet unknown, we have opted here for the conservative suppression genetic interaction definition. This suppressive regulation presumably arises from balanced pgant3/pgant35A function. Consistently, when a single wild-type transgene (UAS-pgant3) is expressed (presynaptic or postsynaptically) in the double mutant (pgant3,pgant35A), the other mutant phenotype (pgant35A) reemerges. Moreover, overexpression of either pgant 3 or pgant $35 \mathrm{~A}$ individually in neuron or muscle decreases neurotransmission strength, which is the opposite consequence of single loss of function. These results reveal a pgant3/ pgant $35 \mathrm{~A}$ suppressive mechanism dependent on the balance between these two genes on both sides of the synapse.

The pgant suppressive mechanism regulates synaptic ultrastructural organization, including presynaptic vesicle pools and PSP size. Like other synaptic phenotypes, PSP size is elevated in single pgant3/pgant $35 \mathrm{~A}$ mutants but normal in double mutants. Importantly, PSP compartments apposed to presynaptic active zones are expanded in trans-synaptic WNT/BMP signaling ligand mutants (Packard et al., 2002; Tian and Ten Hagen, 2007; Ren et al., 2009; Nahm et al., 2010; Kamimura et al., 2013), as well as mutants affecting extracellular HSPG regulators of trans-synaptic signaling (Packard et al., 2002; Tian and Ten Hagen, 2007; Ren et al., 2009; Nahm et al., 2010; Kamimura et al., 2013). Consistently, we identified the trans-synaptic Ten-m/ $\alpha$ PS2 integrin signaling pair (Mosca et al., 2012) to be suppressively regulated by the pgant3/pgant $35 \mathrm{~A}$ mechanism. Ten-m/ $\alpha \mathrm{PS} 2$ integrin interactions are known to drive intercellular adhesion (Graner et al., 1998), and pgant3 is known to regulate integrin-ligand secretion and promote adhesion in the developing Drosophila wing (Zhang et al., 2010). At the Drosophila NMJ, both Ten-m ligand and $\alpha$ PS2 integrin are localized presynaptically and postsynaptically (Mosca et al., 2012). Based on these extensive established interactions, we interpret the enlarged PSP in pgant 3 and pgant $35 \mathrm{~A}$ single mutants to a consequence of impaired Ten-m/integrin signaling. Because the spacing between presynaptic and postsynaptic membranes is not affected and normally apposed presynaptic/ postsynaptic membranes occur with enlarged PSPs, we consider this to be a postsynaptic defect. This is not surprising because $\alpha \mathrm{PS} 2 /$ Ten- $\mathrm{m}$ are both transmembrane proteins, and integrin signaling is well known to bridge to the cytoskeleton (Delon and Brown, 2007). Thus, an enlarged PSP can manifest on the inside of the postsynaptic membrane as a result of impaired integrin signaling. The levels of Ten-m and $\alpha$ PS2, as well as PSP size, are all suppressively regulated by the pgant 3 / pgant 35 A mechanism.
Synaptic O-GalNAc abundance is likewise suppressively regulated by pgant 3 and pgant $35 \mathrm{~A}$, with levels elevated in single mutants and normal in double mutants. Like mammalian pgants (GalNAc-Ts or ppGalNAcTs), Drosophila pgants (12 total) are thought to function hierarchically, competing for naked or glycosylated substrates to regulate final O-GalNAc density (Ten Hagen et al., 2003a). The observed suppressive mechanism suggests that pgant 3 and pgant $35 \mathrm{~A}$ may function at the same tier of glycosylation. Alternatively, with the imbalance induced by pgant mutations, other pgant family members may be dysregulated, leading to increased O-GalNAc synaptic glycosylation. Normally, Golgi-resident pgants relocated to the ER are known to increase O-GalNAc glycosylation (Gill et al., 2010), dependent on Src activation downstream of integrin signaling (Mitra and Schlaepfer, 2006), which is misregulated in pgant mutants. In addition to well described $\alpha / \beta$ integrins functions at the mammalian NMJ, $\alpha 3$ integrin affects hippocampal dendrite stability and function (Kerrisk et al., 2013), whereas $\beta 3$ integrin associates with GluA2 AMPA receptors (Pozo et al., 2012). In Drosophila, we have shown that $\alpha \mathrm{PS} 1-\alpha \mathrm{PS} 13$ and $\beta \mathrm{PS}$ regulate synapse assembly and neurotransmission strength (Beumer et al., 1999; Rohrbough et al., 2000), agreeing with pgant roles shown here in the presynaptic vesicle pool and postsynaptic glutamate receptor regulation. In synaptic plasticity, $\alpha 3 / 5 / 8$ and $\beta 1$ integrin knockdown all impair hippocampal long-term potentiation (Chan et al., 2003, 2006). Similarly, Drosophila $\alpha \mathrm{PS} 3$ (Volado) and $\beta \mathrm{PS}$ mutants show impaired augmentation and PTP (Rohrbough et al., 2000), agreeing with pgant roles shown here in maintaining both plasticity phases. In addition to the joint Ten-m $/ \alpha$ PS2 downregulation in pgant 3 and pgant $35 \mathrm{~A}$, each mutant also displays distinct misregulation of integrin signaling components $\left(\beta_{\mathrm{v}}\right.$ and $\beta \mathrm{PS}$, respectively), with roles in neurotransmission and synaptic plasticity (Rohrbough et al., 2000; Tsai et al., 2012a; Tran and Ten Hagen, 2013).

All phases of synaptic plasticity (facilitation, augmentation, and potentiation) are suppressively regulated by pgant 3 and pgant35A. To investigate mechanisms of these activitydependent changes, we used optogenetic stimulation to test acute subcellular ultrastructure and integrin signaling effects (Fenno et al., 2011). Classical studies coupling traditional electrical nerve stimulation to ultrastructural analysis at frog NMJ revealed dynamic vesicle fusion after single stimuli (Heuser and Reese, 1981) and vesicle depletion after a prolonged train of $10 \mathrm{~Hz}$ stimulation (Ceccarelli et al., 1972). Recent studies using channelrhodopsin (ChIEF) optogenetic stimulation identified an ultrafast endocytic mechanism at the Caenorhabditis elegans NMJ (Watanabe et al., 2013a), which was subsequently validated in hippocampal synapses (Watanabe et al., 2013b), but did not assay effects on vesicle pools. Using the same ChIEF optogenetic tool in Drosophila, we find that a brief, high-frequency light train $(10 \mathrm{~Hz}, 60 \mathrm{~ms}$ pulses for $60 \mathrm{~s}$ ) drives a depression of vesicles in distinct pools around presynaptic active zones. We also find activity-dependent expansion of PSPs in controls, which fails in both pgant single mutants but is restored in double mutants, again showing a suppressive mechanism. Consistently, we identify suppressive activitydependent elevation of integrin downstream Talin signaling in only control and double mutant conditions, supported by known roles of Talin-mediated $\alpha$ PS2 integrin signaling (Devenport et al., 2007). Moreover, we find a lack of activity-dependent pFAK regulation, supported by previous studies showing activitydependent decreases in pFAK signaling at the Drosophila NMJ (Tsai et al., 2012a). Importantly, RGD treatment perturbing integrin signaling and synaptic plasticity also alters synaptic pFAK 
levels (Stäubli et al., 1998; Rohrbough et al., 2000; Russo et al., 2013). Consistent with this mechanism, RGD treatment acts synergistically with pgant mutations to prevent the manifestation of a synaptic plasticity.

In summary, this is the first investigation of synaptic pgant roles, which combines molecular, electrophysiological, electron microscopy, and optogenetic approaches. We identify here a novel suppressive mechanism between two pgant family members ( pgant3 and pgant35A) regulating synaptomatrix O-GalNAc glycosylation state, coupled presynaptic active zone and postsynaptic glutamate receptor assembly, transmission strength, integrin signaling and postsynaptic adhesion, and the appearance of activity-dependent plasticity. Future studies will seek to determine whether $\mathrm{Ca}^{2+}$ and/or CaMKII signaling mechanisms (Tsai et al., 2012a) are misregulated during pgant synaptic dysfunction, as the leading causal link between activity and observed synaptic changes. Based on recent reports that show that O-GalNac levels regulate proteolytic cleavage and ligand secretion (Zhang et al., 2014), we will test whether the pgant suppressive mechanism may reflect interactions between additional pgants or within other enzymatic classes. A final priority will be investigation of pgantmediated regulation of disease-related synaptic proteins, including Dystroglycan (Henry et al., 2001) and Neurofimbrin (Tsai et al., 2012b), to test the hypothesis that heritable neurological and neuromuscular disorders are causally related to the pgant synaptic glycan mechanism.

\section{References}

Bahr BA, Staubli U, Xiao P, Chun D, Ji ZX, Esteban ET, Lynch G (1997) Arg-Gly-Asp-Ser-selective adhesion and the stabilization of long-term potentiation: pharmacological studies and the characterization of a candidate matrix receptor. J Neurosci 17:1320-1329. Medline

Beumer KJ, Rohrbough J, Prokop A, Broadie K (1999) A role for PS integrins in morphological growth and synaptic function at the postembryonic neuromuscular junction of Drosophila. Development 126:5833-5846. Medline

Beumer K, Matthies HJG, Bradshaw A, Broadie K (2002) Integrins regulate DLG/FAS2 via a CaM kinase II-dependent pathway to mediate synapse elaboration and stabilization during postembryonic development. Development 129:3381-3391. Medline

Brand AH, Perrimon N (1993) Targeted gene expression as a means of altering cell fates and generating dominant phenotypes. Development 118 : 401-415. Medline

Broadie K, Baumgartner S, Prokop A (2011) Extracellular matrix and its receptors in Drosophila neural development. Dev Neurobiol 71:11021130. CrossRef Medline

Brower DL, Wilcox M, Piovant M, Smith RJ, Reger LA (1984) Related cellsurface antigens expressed with positional specificity in Drosophila imaginal discs. Proc Natl Acad Sci U S A 81:7485-7489. CrossRef Medline

Ceccarelli B, Hurlbut WP, Mauro A (1972) Depletion of vesicles from frog neuromuscular junctions by prolonged tetanic stimulation. J Cell Biol 54:30-38. CrossRef Medline

Chan CS, Weeber EJ, Kurup S, Sweatt JD, Davis RL (2003) Integrin requirement for hippocampal synaptic plasticity and spatial memory. J Neurosci 23:7107-7116. Medline

Chan CS, Weeber EJ, Zong L, Fuchs E, Sweatt JD, Davis RL (2006) $\beta 1$ integrins are required for hippocampal AMPA receptor-dependent synaptic transmission, synaptic plasticity, and working memory. J Neurosci 26:223-232. CrossRef Medline

Chia J, Tham KM, Gill DJ, Bard-Chapeau EA, Bard FA (2014) ERK8 is a negative regulator of $\mathrm{O}-\mathrm{GalNAc}$ glycosylation and cell migration. Elife 3:e01828. CrossRef Medline

Chintapalli VR, Wang J, Dow JAT (2007) Using FlyAtlas to identify better Drosophila melanogaster models of human disease. Nat Genet 39:715720. CrossRef Medline

Dani N, Broadie K (2012) Glycosylated synaptomatrix regulation of transsynaptic signaling. Dev Neurobiol 72:2-21. CrossRef Medline

Dani N, Nahm M, Lee S, Broadie K (2012) A targeted glycan-related gene screen reveals heparan sulfate proteoglycan sulfation regulates WNT and BMP trans-synaptic signaling. PLoS Genet 8:e1003031. CrossRef Medline

Delon I, Brown NH (2007) Integrins and the actin cytoskeleton. Curr Opin Cell Biol 19:43-50. CrossRef Medline

Devenport D, Bunch TA, Bloor JW, Brower DL, Brown NH (2007) Mutations in the Drosophila $\alpha$ PS2 integrin subunit uncover new features of adhesion site assembly. Dev Biol 308:294-308. CrossRef Medline

Dityatev A, Schachner M (2003) Extracellular matrix molecules and synaptic plasticity. Nat Rev Neurosci 4:456-468. CrossRef Medline

Featherstone DE, Rushton E, Rohrbough J, Liebl F, Karr J, Sheng Q, Rodesch CK, Broadie K (2005) An essential Drosophila glutamate receptor subunit that functions in both central neuropil and neuromuscular junction. J Neurosci 25:3199-3208. CrossRef Medline

Fenno L, Yizhar O, Deisseroth K (2011) The development and application of optogenetics. Annu Rev Neurosci 34:389-412. CrossRef Medline

Fogerty FJ, Fessler LI, Bunch TA, Yaron Y, Parker CG, Nelson RE, Brower DL, Gullberg D, Fessler JH (1994) Tiggrin, a novel Drosophila extracellular matrix protein that functions as a ligand for Drosophila alpha PS2 beta PS integrins. Development 120:1747-1758. Medline

Freeze HH (2006) Genetic defects in the human glycome. Nat Rev Genet 7:537-551. CrossRef Medline

Gagneux P, Varki A (1999) Evolutionary considerations in relating oligosaccharide diversity to biological function. Glycobiology 9:747-755. CrossRef Medline

Gill DJ, Chia J, Senewiratne J, Bard F (2010) Regulation of O-glycosylation through Golgi-to-ER relocation of initiation enzymes. J Cell Biol 189: 843-858. CrossRef Medline

Graner MW, Bunch TA, Baumgartner S, Kerschen A, Brower DL (1998) Splice variants of the Drosophila PS2 integrins differentially interact with RGD-containing fragments of the extracellular proteins tiggrin, Ten-m, and D-laminin 2. J Biol Chem 273:18235-18241. CrossRef Medline

Gruntman E, Turner GC (2013) Integration of the olfactory code across dendritic claws of single mushroom body neurons. Nat Neurosci 16 : 1821-1829. CrossRef Medline

Henry MD, Satz JS, Brakebusch C, Costell M, Gustafsson E, Fässler R, Campbell KP (2001) Distinct roles for dystroglycan, betal integrin and perlecan in cell surface laminin organization. J Cell Sci 114:1137-1144. Medline

Heuser JE, Reese TS (1981) Structural changes after transmitter release at the frog neuromuscular junction. J Cell Biol 88:564-580. CrossRef Medline

Honts JE, Sandrock TS, Brower SM, O’Dell JL, Adams AE (1994) Actin mutations that show suppression with fimbrin mutations identify a likely fimbrin-binding site on actin. J Cell Biol 126:413-422. CrossRef Medline

Inoue Y, Hayashi S (2007) Tissue-specific laminin expression facilitates integrin-dependent association of the embryonic wing disc with the trachea in Drosophila. Dev Biol 304:90-101. CrossRef Medline

Kamimura K, Ueno K, Nakagawa J, Hamada R, Saitoe M, Maeda N (2013) Perlecan regulates bidirectional Wnt signaling at the Drosophila neuromuscular junction. J Cell Biol 200:219-233. CrossRef Medline

Kerrisk ME, Greer CA, Koleske AJ (2013) Integrin $\alpha 3$ is required for late postnatal stability of dendrite arbors, dendritic spines and synapses, and mouse behavior. J Neurosci 33:6742-6752. CrossRef Medline

Keshishian H, Broadie K, Chiba A, Bate M (1996) The drosophila neuromuscular junction: a model system for studying synaptic development and function. Annu Rev Neurosci 19:545-575. CrossRef Medline

Levine A, Bashan-Ahrend A, Budai-Hadrian O, Gartenberg D, Menasherow S, Wides R (1994) Odd Oz: a novel Drosophila pair rule gene. Cell 77: 587-598. CrossRef Medline

Lin DM, Goodman CS (1994) Ectopic and increased expression of Fasciclin II alters motoneuron growth cone guidance. Neuron 13:507-523. CrossRef Medline

Long AA, Kim E, Leung HT, Woodruff E 3rd, An L, Doerge RW, Pak WL, Broadie K (2008) Presynaptic calcium channel localization and calcium-dependent synaptic vesicle exocytosis regulated by the Fuseless protein. J Neurosci 28:3668-3682. CrossRef Medline

Martin D, Zusman S, Li X, Williams EL, Khare N, DaRocha S, ChiquetEhrismann R, Baumgartner S (1999) wing blister, a new Drosophila laminin alpha chain required for cell adhesion and migration during embryonic and imaginal development. J Cell Biol 145:191-201. CrossRef Medline

Mitra SK, Schlaepfer DD (2006) Integrin-regulated FAK-Src signaling in 
normal and cancer cells. Curr Opin Cell Biol 18:516-523. CrossRef Medline

Mosca TJ, Hong W, Dani VS, Favaloro V, Luo L (2012) Trans-synaptic Teneurin signalling in neuromuscular synapse organization and target choice. Nature 484:237-241. CrossRef Medline

Nahm M, Long AA, Paik SK, Kim S, Bae YC, Broadie K, Lee S (2010) The Cdc42-selective GAP rich regulates postsynaptic development and retrograde BMP transsynaptic signaling. J Cell Biol 191:661-675. CrossRef Medline

Ohtsubo K, Marth JD (2006) Glycosylation in cellular mechanisms of health and disease. Cell 126:855-867. CrossRef Medline

Packard M, Koo ES, Gorczyca M, Sharpe J, Cumberledge S, Budnik V (2002) The Drosophila Wnt, wingless, provides an essential signal for pre- and postsynaptic differentiation. Cell 111:319-330. CrossRef Medline

Parkhomovskiy N, Kammesheidt A, Martin PT (2000) N-acetyllactosamine and the CT carbohydrate antigen mediate agrin-dependent activation of MuSK and acetylcholine receptor clustering in skeletal muscle. Mol Cell Neurosci 15:380-397. CrossRef Medline

Parkinson W, Dear ML, Rushton E, Broadie K (2013) N-glycosylation requirements in neuromuscular synaptogenesis. Development 140:49704981. CrossRef Medline

Pozo K, Cingolani LA, Bassani S, Laurent F, Passafaro M, Goda Y (2012) $\beta 3$ integrin interacts directly with GluA2 AMPA receptor subunit and regulates AMPA receptor expression in hippocampal neurons. Proc Natl Acad Sci U S A 109:1323-1328. CrossRef Medline

Ren Y, Kirkpatrick CA, Rawson JM, Sun M, Selleck SB (2009) Cell typespecific requirements for heparan sulfate biosynthesis at the Drosophila neuromuscular junction: effects on synapse function, membrane trafficking, and mitochondrial localization. J Neurosci 29:8539-8550. CrossRef Medline

Repnikova E, Koles K, Nakamura M, Pitts J, Li H, Ambavane A, Zoran MJ, Panin VM (2010) Sialyltransferase regulates nervous system function in Drosophila. J Neurosci 30:6466-6476. CrossRef Medline

Rohrbough J, Grotewiel MS, Davis RL, Broadie K (2000) Integrin-mediated regulation of synaptic morphology, transmission, and plasticity. J Neurosci 20:6868-6878. Medline

Rohrbough J, Rushton E, Woodruff E 3rd, Fergestad T, Vigneswaran K, Broadie K (2007) Presynaptic establishment of the synaptic cleft extracellular matrix is required for post-synaptic differentiation. Genes Dev 21:2607-2628. CrossRef Medline

Rushton E, Rohrbough J, Broadie K (2009) Presynaptic secretion of mindthe-gap organizes the synaptic extracellular matrix-integrin interface and postsynaptic environments. Dev Dyn 238:554-571. CrossRef Medline

Russo MA, Paolillo M, Sanchez-Hernandez Y, Curti D, Ciusani E, Serra M, Colombo L, Schinelli S (2013) A small-molecule RGD-integrin antagonist inhibits cell adhesion, cell migration and induces anoikis in glioblastoma cells. Int J Oncol 42:83-92. CrossRef Medline

Schwientek T, Bennett EP, Flores C, Thacker J, Hollmann M, Reis CA, Behrens J, Mandel U, Keck B, Schäfer MA, Haselmann K, Zubarev R, Roepstorff P, Burchell JM, Taylor-Papadimitriou J, Hollingsworth MA, Clausen H (2002) Functional conservation of subfamilies of putative UDP-N-acetylgalactosamine:polypeptide $\mathrm{N}$-acetylgalactosaminyltransferases in Drosophila, Caenorhabditis elegans, and mammals. One subfamily composed of 1(2)35Aa is essential in Drosophila. J Biol Chem 277: 22623-22638. CrossRef Medline

Scott H, Panin VM (2014) The role of protein N-glycosylation in neural transmission. Glycobiology 24:407-417. CrossRef Medline

Stäubli U, Chun D, Lynch G (1998) Time-dependent reversal of long-term potentiation by an integrin antagonist. J Neurosci 18:3460-3469. Medline

Subramanian A, Wayburn B, Bunch T, Volk T (2007) Thrombospondinmediated adhesion is essential for the formation of the myotendinous junction in Drosophila. Development 134:1269-1278. CrossRef Medline

Ten Hagen KG, Tran DT (2002) A UDP-GalNAc:polypeptide N-acetylgalactosaminyltransferase is essential for viability in Drosophila melanogaster. J Biol Chem 277:22616-22622. CrossRef Medline
Ten Hagen KG, Fritz TA, Tabak LA (2003a) All in the family: the UDPGalNAc:polypeptide N-acetylgalactosaminyltransferases. Glycobiology 13:1R-16R. CrossRef Medline

Ten Hagen KG, Tran DT, Gerken TA, Stein DS, Zhang Z (2003b) Functional characterization and expression analysis of members of the UDPGalNAc:polypeptide N-acetylgalactosaminyltransferase family from Drosophila melanogaster. J Biol Chem 278:35039-35048. CrossRef Medline

Tian E, Ten Hagen KG (2006) Expression of the UDP-GalNAc: polypeptide $\mathrm{N}$-acetylgalactosaminyltransferase family is spatially and temporally regulated during Drosophila development. Glycobiology 16:83-95. CrossRef Medline

Tian E, Ten Hagen KG (2007) A UDP-GalNAc:polypeptide N-acetylgalactosaminyltransferase is required for epithelial tube formation. J Biol Chem 282:606-614. CrossRef Medline

Tran DT, Ten Hagen KG (2013) Mucin-type O-glycosylation during development. J Biol Chem 288:6921-6929. CrossRef Medline

Tsai PI, Wang M, Kao HH, Cheng YJ, Lin YJ, Chen RH, Chien CT (2012a) Activity-dependent retrograde laminin A signaling regulates synapse growth at Drosophila neuromuscular junctions. Proc Natl Acad Sci U S A 109:17699-17704. CrossRef Medline

Tsai PI, Wang M, Kao HH, Cheng YJ, Walker JA, Chen RH, Chien CT (2012b) Neurofibromin mediates FAK signaling in confining synapse growth at Drosophila neuromuscular junctions. J Neurosci 32:1697116981. CrossRef Medline

Wagh DA, Rasse TM, Asan E, Hofbauer A, Schwenkert I, Dürrbeck H, Buchner S, Dabauvalle MC, Schmidt M, Qin G, Wichmann C, Kittel R, Sigrist SJ, Buchner E (2006) Bruchpilot, a protein with homology to ELKS/ CAST, is required for structural integrity and function of synaptic active zones in Drosophila. Neuron 49:833-844. CrossRef Medline

Wallace BG (1990) Inhibition of agrin-induced acetylcholine-receptor aggregation by heparin, heparan sulfate, and other polyanions. J Neurosci 10:3576-3582. Medline

Wang K, Liu Y, Li Y, Guo Y, Song P, Zhang X, Zeng S, Wang Z (2011) Precise spatiotemporal control of optogenetic activation using an acousto-optic device. PLoS One 6:e28468. CrossRef Medline

Watanabe S, Liu Q, Davis MW, Hollopeter G, Thomas N, Jorgensen NB, Jorgensen EM (2013a) Ultrafast endocytosis at Caenorhabditis elegans neuromuscular junctions. Elife 2:e00723. CrossRef Medline

Watanabe S, Rost BR, Camacho-Pérez M, Davis MW, Söhl-Kielczynski B, Rosenmund C, Jorgensen EM (2013b) Ultrafast endocytosis at mouse hippocampal synapses. Nature 504:242-247. CrossRef Medline

Wodarz A, Hinz U, Engelbert M, Knust E (1995) Expression of crumbs confers apical character on plasma membrane domains of ectodermal epithelia of Drosophila. Cell 82:67-76. CrossRef Medline

Yee GH, Hynes RO (1993) A novel, tissue-specific integrin subunit, beta nu, expressed in the midgut of Drosophila melanogaster. Development 118: 845-858. Medline

Yoshida H, Fuwa TJ, Arima M, Hamamoto H, Sasaki N, Ichimiya T, Osawa K, Ueda R, Nishihara S (2008) Identification of the Drosophila core 1 beta1,3-galactosyltransferase gene that synthesizes $\mathrm{T}$ antigen in the embryonic central nervous system and hemocytes. Glycobiology 18:10941104. CrossRef Medline

Zhang L, Ten Hagen KG (2011) The cellular microenvironment and cell adhesion: a role for O-glycosylation. Biochem Soc Trans 39:378-382. CrossRef Medline

Zhang L, Zhang Y, Hagen KG (2008) A mucin-type O-glycosyltransferase modulates cell adhesion during Drosophila development. J Biol Chem 283:34076-34086. CrossRef Medline

Zhang L, Tran DT, Ten Hagen KG (2010) An O-glycosyltransferase promotes cell adhesion during development by influencing secretion of an extracellular matrix integrin ligand. J Biol Chem 285:19491-19501. CrossRef Medline

Zhang L, Syed ZA, van Dijk Härd I, Lim JM, Wells L, Ten Hagen KG (2014) O-glycosylation regulates polarized secretion by modulating Tangol stability. Proc Natl Acad Sci U S A 111:7296-7301. CrossRef Medline 